\title{
STABILITY OF POLE SOLUTIONS FOR PLANAR PROPAGATING FLAMES: II. PROPERTIES OF EIGENVALUES/EIGENFUNCTIONS AND IMPLICATIONS TO STABILITY*
}

\author{
DIMITRI VAYNBLAT ${ }^{\dagger}$ AND MOSHE MATALON $^{\dagger}$
}

\begin{abstract}
In a previous paper (Part I) we focused our attention on pole solutions that arise in the context of flame propagation. The nonlinear development that follows after a planar flame front becomes unstable is described by a single nonlinear PDE which admits pole solutions as equilibrium states. Specifically, we were concerned with coalescent steady states, which correspond to steadily propagating single-peak structures extended periodically over the infinite domain. This pattern is one that is commonly observed in experiments. In order to examine the linear stability of these equilibrium solutions, we formulated in Part I the corresponding eigenvalue problem and derived exact analytical expressions for the spectrum and the corresponding eigenfunctions. In this paper, we examine their properties as they relate to the stability issue. Being based on analytical expressions, our results resolve earlier controversies that resulted from numerical investigations of the stability problem. We show that, for any period $2 L$, there always exists one and only one stable steady coalescent pole solution. We also examine the dependence of the eigenvalues and eigenfunctions on $L$ which provides insight into the behavior of the nonlinear PDE and, consequently, on the nonlinear dynamics of the flame front.
\end{abstract}

Key words. flame front, pole decomposition, coalescent pole solution, stability, eigenvalues and eigenfunctions

AMS subject classifications. 76E99, 35Q35, 80A32

PII. S0036139998346440

1. Introduction. In a previous paper [1], that we shall refer to as Part I, we were concerned with the periodic solutions of the nonlinear PDE

$$
\varphi_{t}=\frac{1}{2} I\{\varphi ; x\}+\alpha \varphi_{x x}+\frac{1}{2} \varphi_{x}^{2}
$$

over the interval $-L<x<L$. The operator $I\{\cdot ; x\}$ is a linear singular nonlocal operator defined such that $I\{\varphi ; x\}=-\mathcal{H}\left\{\varphi_{x} ; x\right\}$, where $\mathcal{H}\{\cdot ; x\}$ is the Hilbert transform. This equation describes the nonlinear development that follows when a nominally planar flame front becomes unstable [2]. Here $\varphi(x, t)$ denotes the flame front displacement, $x$ is the spatial coordinate, and $t$ is the time. The physicochemical parameter $\alpha$ depends primarily on the gas thermal expansion and is responsible for the Darrieus-Landau instability of the planar front $[3,4]$. As noted in Part I, the problem depends on a single parameter $\tilde{\alpha}=\pi \alpha / L$ which, for convenience, will be used interchangeably with its reciprocal $\gamma=1 / \tilde{\alpha}$. We note that changes in $L$, the parameter used in $[5,6,7,8]$, are proportional to changes in $\gamma$ and inversely proportional to changes in $\tilde{\alpha}$. With this scaling the domain of integration is fixed and equal to $2 \pi$. For further details the reader should consult Part I. Also, in order to minimize duplication, we shall use the notation (I-n.m) when referring to equation (n.m) of Part I; we will state explicitly only the necessary equations.

*Received by the editors October 29, 1998; accepted for publication March 23, 1999; published electronically February 2, 2000. This research was partially supported by the National Science Foundation under grants DMS9703716 and CTS9521022.

http://www.siam.org/journals/siap/60-2/34644.html

${ }^{\dagger}$ Department of Engineering Sciences and Applied Mathematics, McCormick School of Engineering and Applied Science, Northwestern University, Evanston, IL 60208-3125 (matalon@nwum.edu).

${ }^{\ddagger}$ Current address: Applied Mathematics 217-50, Caltech, Pasadena, CA 91125. 
The trivial solution $\varphi=0$ of (1.1) corresponds to the flat flame front, which is known to be unstable for $\gamma>\gamma_{1}=2$. At the same time, for $\gamma>\gamma_{1}$ the nonlinear PDE admits exact pole solutions as equilibrium states. The pole decomposition technique formally reduces the PDE to a finite set of ODEs which describe the motion of the poles in the complex plane. There is a natural tendency, implied by these ODEs, for the poles to align parallel to the imaginary axis. The particular set of solutions thus obtained is referred to as the family of coalescent states, and the set of equilibrium solutions obtained when the poles are time independent is referred to as the family of coalescent steady states. The members of this family are distinguished by the number of poles they possess such that an $N$-pole coalescent steady state $\varphi_{N}$, with $N=0,1, \ldots, \infty$, is a solution made up of $N$ aligned poles. The zero-pole solution corresponds to a flat front. An $N$-pole solution with $N \geq 1$ corresponds to a cusp-like structure that propagates at constant speed without change in its shape. As the value of $\gamma$ increases this solution becomes more singular and develops a real cusp as $\gamma \rightarrow \infty$. For a given $\gamma$ there is an upper bound on the number of poles that a coalescent steady state possesses, namely, $N \leq N_{0}(\gamma)$ with $N_{0}(\gamma)$ given by (I-3.24). For given $\gamma$ and $N \leq N_{0}(\gamma)$ there exists one and only one coalescent steady state. The larger $N$, the larger the peak of the flame front. At the critical point $\gamma=\gamma_{1}$ at which the flat front becomes unstable, the one-pole solution is born from the zero-pole solution. At the critical point $\gamma=\gamma_{2}=6$, the two-pole solution is born out of the one-pole solution. And, in general, the $N$-pole solution bifurcates from the $(N-1)$-pole solution at $\gamma=\gamma_{N}=2(2 N-1)$.

For a coalescent state, a pole can be uniquely identified by its imaginary part. We shall therefore use the notation $y_{n}^{k}$ for both the $n$th pole of the $k$-pole coalescent steady state and the imaginary part of this pole. In physical space the imaginary part represents the depth of the cusp forming on the flame front; the real part, common to all poles, represents the location of the cusp along the front. Without loss of generality it is assumed in this paper that the cusp is located at the origin. In Figure 1.1 we present the dependence of the poles of the coalescent steady states on $\gamma$, as calculated in Part I, in two ways. Each provides a different interpretation: the first is perhaps the more natural one; the second was found more useful in the discussion below.

The first way, best illustrated in Figure 1.1(a), is to consider each solution, $\varphi_{N}$, as being composed of $N$ poles. All but one pole of a coalescent steady state $\varphi_{N}$ bifurcate from the poles of the parent solution $\varphi_{N-1}$; the last pole is born at infinity. Thus, at $\gamma=\gamma_{N}$, the first $N-1$ poles $y_{1}^{N}, \ldots, y_{N-1}^{N}$ bifurcate from the poles $y_{1}^{N-1}, \ldots, y_{N-1}^{N-1}$, respectively, whereas the last pole $y_{N}^{N}$ originates at infinity. We observe that $y_{n}^{N}<$ $y_{n}^{N-1}, n=1, \ldots, N-1$, and that all the poles approach zero as $\gamma \rightarrow \infty$.

The second way, best illustrated in Figure 1.1(b), is to consider each solution, $\varphi_{N}$, as being composed of an infinitely countable number of poles. The first $N$ poles of the $N$-pole solution have finite imaginary parts, i.e., $1 / y_{n}^{N}>0$ for $n=1, \ldots, N$; the remaining " $\infty-N$ " poles have infinite imaginary parts, i.e., $1 / y_{n}^{N} \equiv 0$ for $n=N+$ $1, \ldots, \infty$. We shall call a pole with a finite imaginary part a pole of type I and a pole with an infinite imaginary part a pole of type II. All the poles of a coalescent steady state $\varphi_{N}$ bifurcate from the poles of the parent solution $\varphi_{N-1}$. At the bifurcation point, all but one pole keep the types of their parent poles, whereas the type of the $N$ th pole is the opposite to the type of its parent pole. Thus, the following relations hold between the poles of $\varphi_{N}$ and the poles of $\varphi_{N-1}$ :

(1) All the poles of $\varphi_{N}$ bifurcate from the poles of $\varphi_{N-1}$. In other words, $1 / y_{n}^{N}=$ $1 / y_{n}^{N-1}$ at $\gamma=\gamma_{N}$ for $n=1, \ldots, \infty$. 

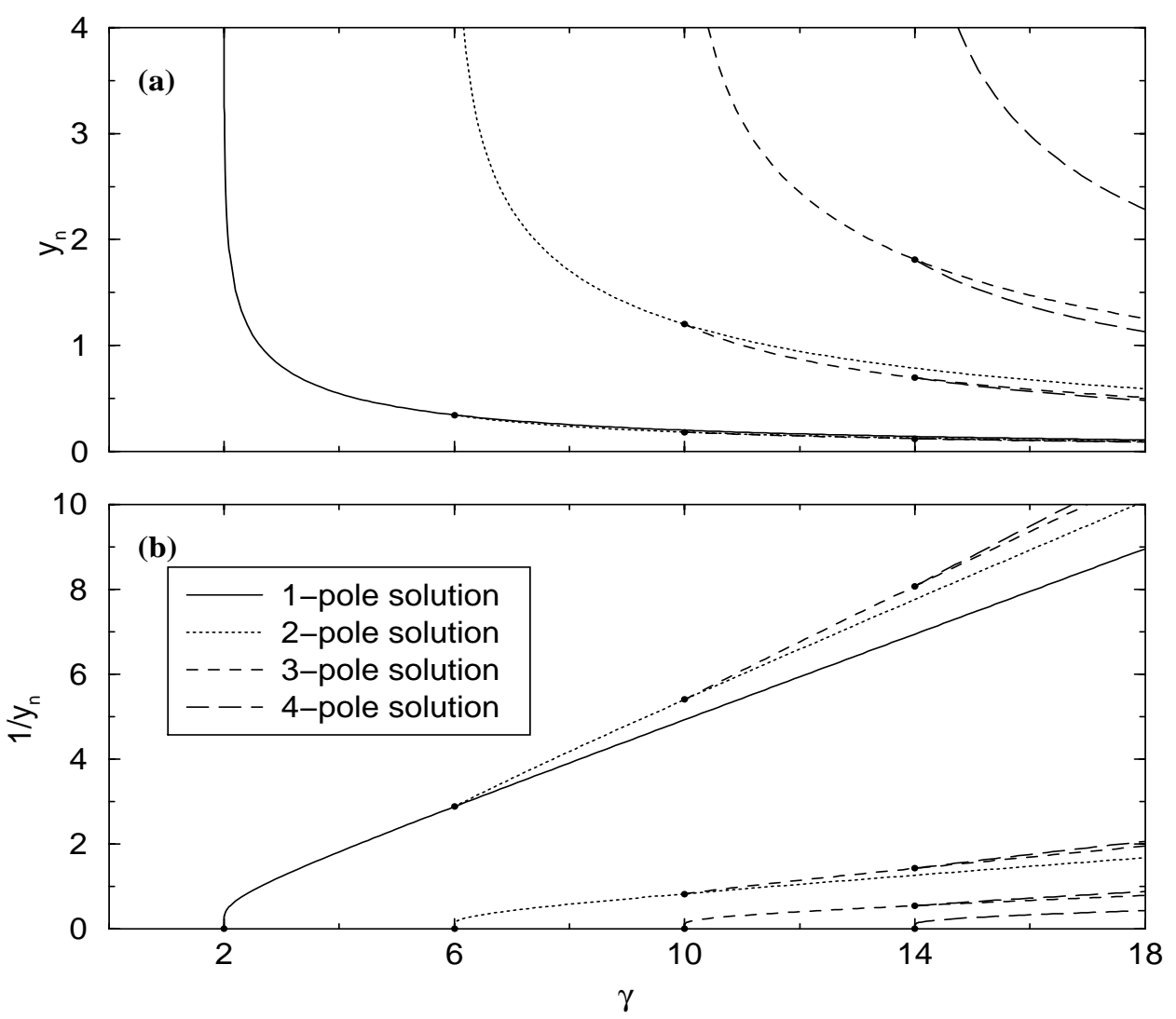

FIG. 1.1. (a) Dependence of the imaginary parts of poles, $y_{n}$, on $\gamma$ for $N$-pole coalescent steady states, $N=1, \ldots, 4$. (b) Dependence of the reciprocals of the imaginary parts of poles, $1 / y_{n}$, on $\gamma$ for $N$-pole coalescent steady states, $N=0, \ldots, 4$. The points where the new poles are born are marked with dots.

(2) The $N$ th pole of $\varphi_{N}$ is of the opposite type from the parent pole, the $N$ th pole of $\varphi_{N-1}$; while $y_{N}^{N-1}$ is of type II, $y_{N}^{N}$ is of type I. In other words, $1 / y_{N}^{N}>1 / y_{N}^{N-1} \equiv 0$ when $\gamma>\gamma_{N}$.

(3) The rest of the poles of $\varphi_{N}$ inherent the types of the parent poles; $y_{n}^{N-1}$ and $y_{n}^{N}$ with $n=1, \ldots, N-1$ are of type I while $y_{n}^{N-1}$ and $y_{n}^{N}$ with $n=N+1, \ldots, \infty$ are of type II. In other words, for $\gamma>\gamma_{N}, 1 / y_{n}^{N}>1 / y_{n}^{N-1}>0$ with $n=1, \ldots, N-1$ and $1 / y_{n}^{N} \equiv 1 / y_{n}^{N-1} \equiv 0$ with $n=N+1, \ldots, \infty$.

Similar to the analyses of $[5,8]$ and $[6,7]$, we are concerned here with the linear stability of the coalescent steady states. In Part I, we formulated the corresponding eigenvalue problem and derived analytical expressions for the spectrum and corresponding eigenfunctions. It was found that the spectrum of the eigenvalue problem associated with an $\mathrm{N}$-pole coalescent steady state consists of two types of eigenvalues.

For type I, there are two sets of eigenvalues, the symmetric set

$$
0>\lambda_{1}^{N, s}>\lambda_{2}^{N, s}>\cdots>\lambda_{N}^{N, s}
$$

and the antisymmetric set

$$
0=\lambda_{1}^{N, a}>\lambda_{2}^{N, a}>\cdots>\lambda_{N}^{N, a} .
$$


All eigenvalues of the symmetric set are distinct from the eigenvalues of the antisymmetric set, for all $\gamma>\gamma_{N}$. Each eigenvalue of type I is a simple eigenvalue; there is only one eigenfunction associated with it. The eigenfunction associated with an eigenvalue of the symmetric set is symmetric with respect to $x=0$; the eigenfunction associated with an eigenvalue of the antisymmetric set is antisymmetric with respect to $x=0$. The eigenfunctions are given in subsection 4.3 of Part I.

For type II, there is only one set that consists of infinitely many eigenvalues, given by

$$
\lambda_{N+S}^{N}=\frac{1}{2} S[1-(S+2 N) 2 \tilde{\alpha}], \quad S=1, \ldots, \infty .
$$

Each eigenvalue of type II has multiplicity two so that there are two different eigenfunctions associated with it - one symmetric and one antisymmetric. The eigenfunctions are given in subsection 4.3 of Part I.

In this paper we show that for any value of the parameter $\gamma$ there exists one and only one asymptotically stable (apart from a trivial lateral translation of the flame front) solution from the family of coalescent steady states. The asymptotically stable solution is the solution with the largest possible (for this particular value of $\gamma$ ) number of poles, $N_{0}(\gamma)$. The same type of bifurcation that takes place at $\gamma_{1}$ occurs at all other bifurcation points: at $\gamma_{1}$ the zero-pole solution becomes unstable while the newborn one-pole solution is stable and, at $\gamma_{N}(N \geq 1)$, the $(N-1)$-pole solution becomes unstable while the newborn $N$-pole solution is stable. Therefore, as the parameter $\gamma$ increases, the equilibrium states of the PDE undergo a cascade of supercritical bifurcations.

We also show that the (linearized) dynamics of poles of type I are linked to the eigenvalues of type I and the (linearized) dynamics of poles of type II are linked to the eigenvalues of type II. As $\gamma$ crosses the bifurcation point $\gamma_{N}$ the following happens: (i) The largest eigenvalue of type II of $\varphi_{N-1}$ (which is the $N$ th eigenvalue) changes its sign from negative to positive. (ii) The $N$ th pole (of type I) of $\varphi_{N}$, born from the $N$ th pole (of type II) of $\varphi_{N-1}$, moves from infinity to a finite location. (iii) The two $N$ th eigenvalues (both of type I, simple) of $\varphi_{N}$, that bifurcated from the $N$ th eigenvalue (of type II, multiplicity two) of $\varphi_{N-1}$, move from zero to negative values. In this way the newborn solution $\varphi_{N}$ gains stability while its parent $\varphi_{N-1}$ becomes unstable.

This paper is organized as follows. In section 2 we examine the properties of the eigenvalues and the eigenfunctions and discuss the implications on the stability of the steady coalescent pole solutions. In section 3 we discuss the links between poles and eigenvalues and the dependence of the solution of the PDE on the parameter $\gamma$. Concluding remarks that include a discussion on directions for future research are presented in section 4 .

2. Spectrum and stability. In this section we examine the properties of the eigenvalues and eigenfunctions and the stability of the steady coalescent pole solutions. As pointed out earlier we assume, without loss of generality, that the common real part of the poles is zero (one needs to set $x_{c}=0$ in all the formulas of subsection 4.3 of Part I).

We denote an eigenfunction in $x$-space by $\psi(x)$ and an eigenfunction in $k$-space as $\breve{\Psi}_{k}$ and adopt the following normalization: arbitrary constants are chosen such that $\sum_{k=1}^{\infty}\left|\check{\Psi}_{k}\right|^{2}=1$ in Fourier-space representation and $\int_{-L}^{L}|\psi(x)|^{2} d x=1$ in physicalspace representation. We note that the quantity $\left|\check{\Psi}_{k}\right|^{2}$ can be thought of as the relative 


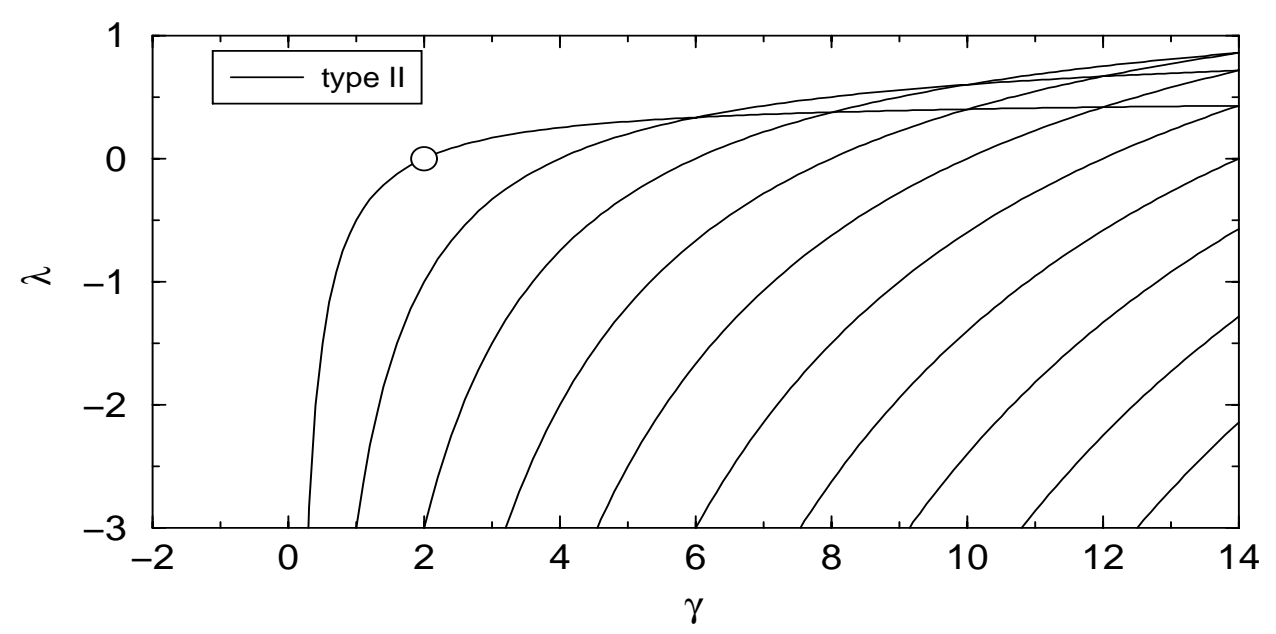

FIG. 2.1. Dependence of the eigenvalues $\lambda$ on $\gamma$ for the zero-pole solution $(N=0)$, or the flat flame front. At $\gamma=2$ the largest eigenvalue crosses the horizontal axis (the point marked with an open circle) and the flat flame becomes unstable.

energy contained in the $k$ th Fourier mode. For further details regarding the notation used, the reader should consult Part I.

We start by examining the zero-pole solution, which corresponds to a steadily propagating flat front.

2.1. Zero-pole solution. When $N=0$, there are no eigenvalues of type I. The eigenvalues of type II,

$$
\lambda_{S}^{0}=\frac{1}{2} S[1-2 \tilde{\alpha} S], \quad S=1, \ldots, \infty,
$$

are plotted in Figure 2.1. Each eigenvalue $\lambda_{S}^{0}$ has multiplicity two; it possesses two eigenfunctions: a pure cosine $\psi_{S}^{0, s}(x)=\cos (\tilde{S} x) / \sqrt{L}$ with wavenumber $\tilde{S}$ and a pure sine $\psi_{S}^{0, a}(x)=\sin (\tilde{S} x) / \sqrt{L}$ with wavenumber $\tilde{S}$. The Fourier-space representation of the eigenfunctions is

$$
\check{\Psi}_{S, k}^{0}= \begin{cases}1 & \text { if } k=S, \\ 0 & \text { otherwise. }\end{cases}
$$

Finally, we point out that the eigenfunctions of the zero-pole solution are independent of $\gamma$.

The linear spectrum (2.1) of the flat front solution implies that there exists a critical value of the parameter $\gamma$, namely, $\gamma_{1}=2$, such that

(1) for $\gamma<\gamma_{1}$ all eigenvalues $\lambda_{S}^{0}$ are negative, the zero-pole solution is asymptotically stable;

(2) at $\gamma=\gamma_{1}$ the first eigenvalue, $\lambda_{1}^{0}$, becomes zero, at this point the zero-pole solution becomes marginally stable;

(3) for $\gamma>\gamma_{1}$ the first eigenvalue, $\lambda_{1}^{0}$, is positive, the zero-pole solution is unstable. 


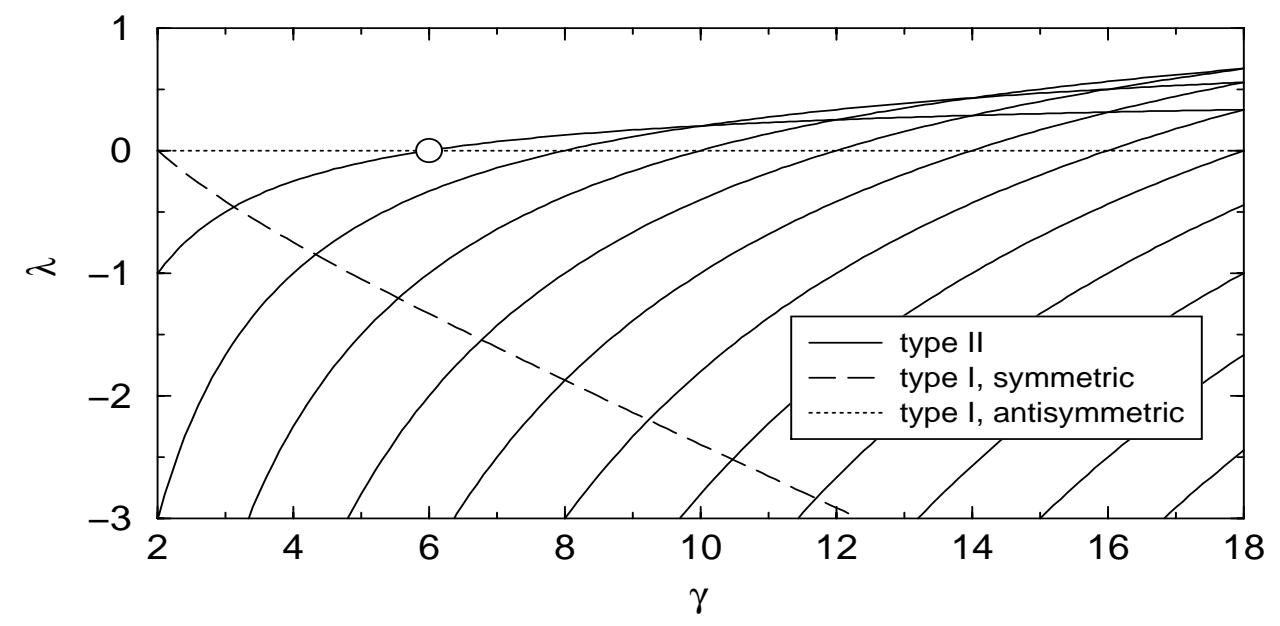

Fig. 2.2. Dependence of the eigenvalues $\lambda$ on $\gamma$ for the one-pole coalescent steady state $(N=1)$ which exists for $\gamma>2$. At $\gamma=6$ the largest eigenvalue crosses the horizontal axis (the point marked with an open circle) and the one-pole coalescent steady state becomes unstable.

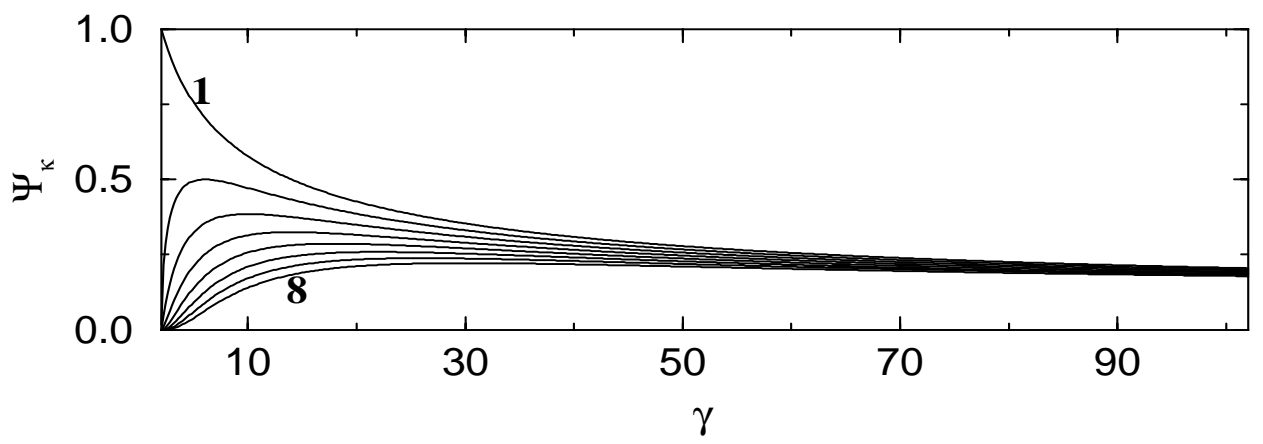

FIG. 2.3. Dependence of the first eight harmonics of the first eigenfunctions (type I) of the one-pole coalescent steady state on $\gamma$.

We have thus recovered the eigenvalues and eigenfunctions of the flat front solution discussed in the introduction. This eigensystem is the only one associated with the PDE (1.1) that, prior to this work, has been known analytically.

Next, we examine the properties of the eigenvalues and eigenfunctions associated with an $N$-pole coalescent steady state $(N>0)$, which corresponds to a steadily propagating cusp-like structure. We start discussing the special cases $N=1,2$ (only $N=2$ is representative of the general case) and then summarize the corresponding properties for the general $N$ case.

2.2. One-pole solution. When $N=1$, there is one symmetric

$$
\lambda_{1}^{1, s}=-\frac{\tilde{\alpha}}{\sinh ^{2} y_{1}}=\sinh (\ln 2 \tilde{\alpha})
$$

and one antisymmetric

$$
\lambda_{1}^{1, a}=0
$$



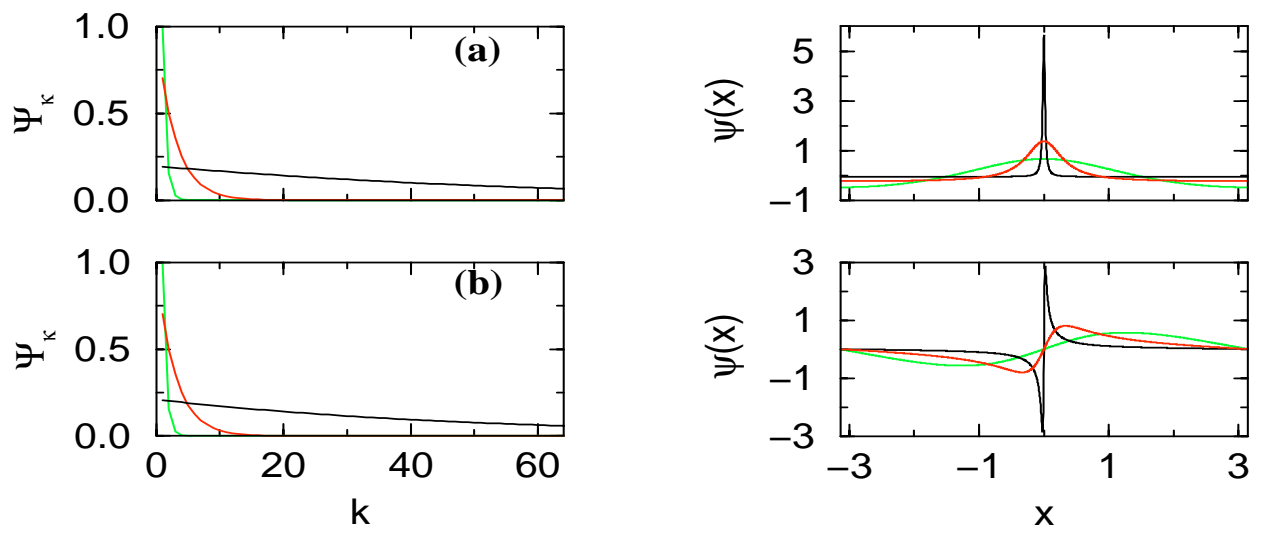

FIG. 2.4. $k$-space representation (left column) and $x$-space representation (right column) of the first (type I) symmetric (row (a)) and antisymmetric (row (b)) eigenfunctions of the one-pole coalescent steady state, at different values of the parameter $\gamma: \gamma=2.1$ - the lightest lines; $\gamma=6.1$ the lines of medium darkness; $\gamma=100.1$ - the darkest lines.

eigenvalue of type I. These eigenvalues are plotted in Figure 2.2. Either eigenvalue is simple. The corresponding eigenfunctions have identical representations in $k$-space, given by

$$
\check{\Psi}_{1, k}^{1}= \begin{cases}\sqrt{\frac{2}{\beta+1}} & \text { if } k=1, \\ \sqrt{\frac{2}{\beta+1}}\left(\frac{\beta-1}{\beta+1}\right)^{(k-1) / 2} & \text { otherwise }\end{cases}
$$

where $\beta=\tilde{\alpha}_{1} / \tilde{\alpha}=\gamma / \gamma_{1}$. The eigenfunctions depend strongly on the parameter $\gamma$. The principal trend in the development of the eigenfunctions of type I, as $\gamma$ increases from $\gamma_{1}$ to $\infty$, is to become more singular.

One observes in Figure 2.3 that the magnitude of the first Fourier harmonic, $\check{\Psi}_{1,1}^{1}$, decreases monotonically from 1 to 0 , while the magnitude of any other harmonic, $\check{\Psi}_{1, k}^{1}$ with $k \geq 2$, first increases from 0 , reaches its maximum value $\sqrt{k^{-k}(k-1)^{(k-1)}}$ at $\gamma=\gamma_{k}$, and then decreases back asymptotically to 0 . Accordingly, one observes (Figure 2.4, left column) the following changes in the profiles of the eigenfunctions in $k$-space, as $\gamma$ increases: The energy concentrated at $k=1$, for $\gamma=\gamma_{1}$, is redistributed in such a way that, as $\gamma$ becomes large, all harmonics become equally significant. The energy associated with any wavenumber $k$ decreases and becomes vanishingly small as $\gamma \rightarrow \infty$. In $x$-space (Figure 2.4, right column) the eigenfunctions become more and more "concentrated" near the origin. For the symmetric eigenfunction, which is a pure cosine at $\gamma_{1}$, the width of the bell-shaped profile decreases while its height increases. In the limit $\gamma \rightarrow \infty$, the height becomes infinitely large and the width vanishingly small; we shall refer to this shape as a "spike." For the antisymmetric eigenfunction, which is a pure sine at $\gamma_{1}$, a pair of spikes, pointing in opposing directions, develop near and on either side of the origin.

There are infinitely many eigenvalues of type II given by formula (1.4), where $N=1$, and plotted in Figure 2.2. Each eigenvalue of type II has multiplicity two. Thus, each possesses two eigenfunctions - one symmetric and one antisymmetric. 



FIG. 2.5. $k$-space representation (left column) and $x$-space representation (right column) of the fourth symmetric eigenfunction (type II) of the one-pole coalescent steady state at different values of the parameter $\gamma$ : (a) $\gamma=2.1$, (b) $\gamma=4.8$, (c) $\gamma=12.0$, (d) $\gamma=23.8$, and (e) $\gamma=100.1$.

The eigenfunctions depend strongly on the parameter $\gamma$. The principal trend in the development of the $m$ th eigenfunctions of type II $(m=2, \ldots, \infty)$, as $\gamma$ increases from $\gamma_{1}$ to $\infty$, is the transfer of energy from the mth Fourier harmonic to the $(m-1)$ th Fourier harmonic: At $\gamma=\gamma_{1}$, the symmetric eigenfunction of type II, $\psi_{m}^{1, s}$, bifurcates from $\psi_{m}^{0, s}$ which is a pure cosine with wavenumber $m$; the antisymmetric eigenfunction of type II, $\psi_{m}^{1, a}$, bifurcates from $\psi_{m}^{0, a}$ which is a pure sine with wavenumber $m$. In the limit $\gamma \rightarrow \infty$, the eigenfunction $\psi_{m}^{1, s}$ asymptotically approaches $\psi_{m-1}^{0, s}$ which is a pure cosine with the wavenumber $m-1$; the eigenfunction $\psi_{m}^{1, a}$ asymptotically approaches $\psi_{m-1}^{0, a}$ which is a pure sine with the wavenumber $m-1$. This transfer of energy does not occur directly, but in three stages as illustrated in Figures 2.52.7 for the case $m=4$. The development of the eigenfunction profiles in $k$ - and $x$-spaces are shown in the graphs (a)-(e) of Figures 2.5-2.6. During the first stage, when values of $\gamma$ are close to $\gamma_{1}$, the energy, initially contained in the $m$ th Fourier mode, is redistributed over a large range of wavenumbers. In $k$-space representation the maximum of the $m$ th eigenfunction, initially located at $k=m$ (a), becomes less pronounced and the contribution of the lower wavenumbers becomes more important. When the maximum reaches $k=1$ (b), the maximum has lost most of its height and a broad range of relatively significant wavenumbers has developed. Accordingly, 

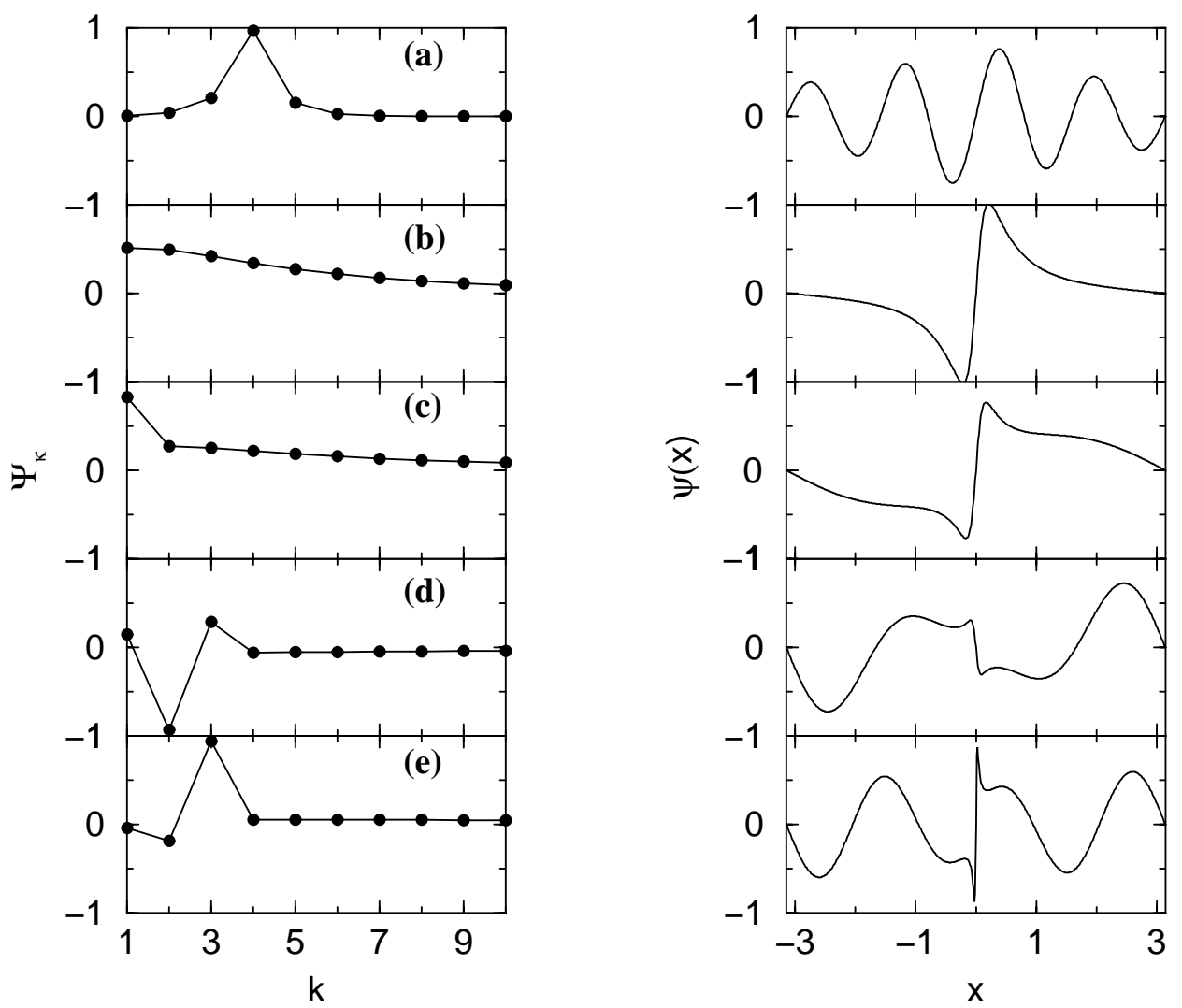

FIG. 2.6. $k$-space representation (left column) and $x$-space representation (right column) of the fourth antisymmetric eigenfunction (type II) of the one-pole coalescent steady state at different values of the parameter $\gamma$ : (a) $\gamma=2.1$, (b) $\gamma=9.1$, (c) $\gamma=12.6$, (d) $\gamma=23.8$, and (e) $\gamma=100.1$.

in $x$-space, the $m$ th eigenfunction is stretched out such that most of the oscillations disappear leaving behind nearly smooth tails with a localized structure in the vicinity of the origin; near the origin the profile of the symmetric eigenfunction is bell shaped and the profile of the antisymmetric eigenfunction is "double bell"-shaped (a pair of bells placed in the opposite sides of the $x$-axis). During the intermediate stage, part of the energy contained in the broad range of harmonics goes into the first wavenumber (c), and then from the first wavenumber into the second wavenumber (d), from the second wavenumber into the third wavenumber, etc., ending up, in general, in the $(m-1)$ th wavenumber. During the last stage, as $\gamma$ becomes large, the $(m-1)$ th wavenumber drains the energy from all the other wavenumbers so that when $\gamma \rightarrow \infty$ the amplitude of the $(m-1)$ th harmonic approaches 1 while the amplitudes of all other harmonics decrease to zero (e). This development is clearly seen in Figure 2.7, where the continuous dependence of the eigenfunctions on $\gamma$ is illustrated for the first eight harmonics, $\check{\Psi}_{4, k}^{1}$ with $k=1, \ldots, 8$; while most of the energy is initially contained in the $m$ th harmonic, it is transferred in stages to the $(m-1)$ th wavenumber as $\gamma$ becomes large.

2.3. Two-pole solution. When $N=2$, there are two symmetric and two antisymmetric eigenvalues of type I. Each eigenvalue of type I is simple. One of the 

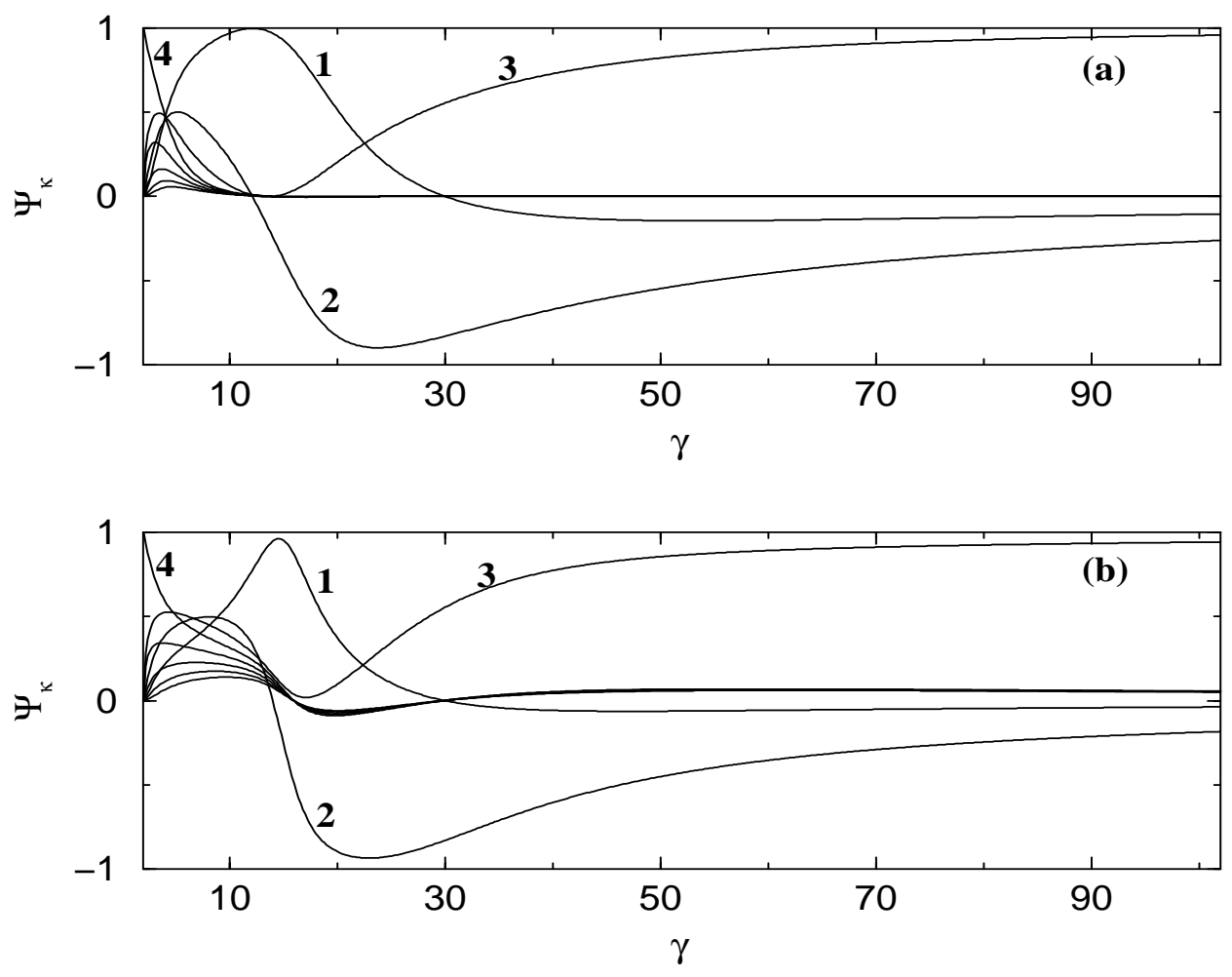

FIG. 2.7. Dependence of the first eight harmonics of the fourth symmetric (a) and antisymmetric (b) eigenfunctions (type II) of the one-pole coalescent steady state on $\gamma$.

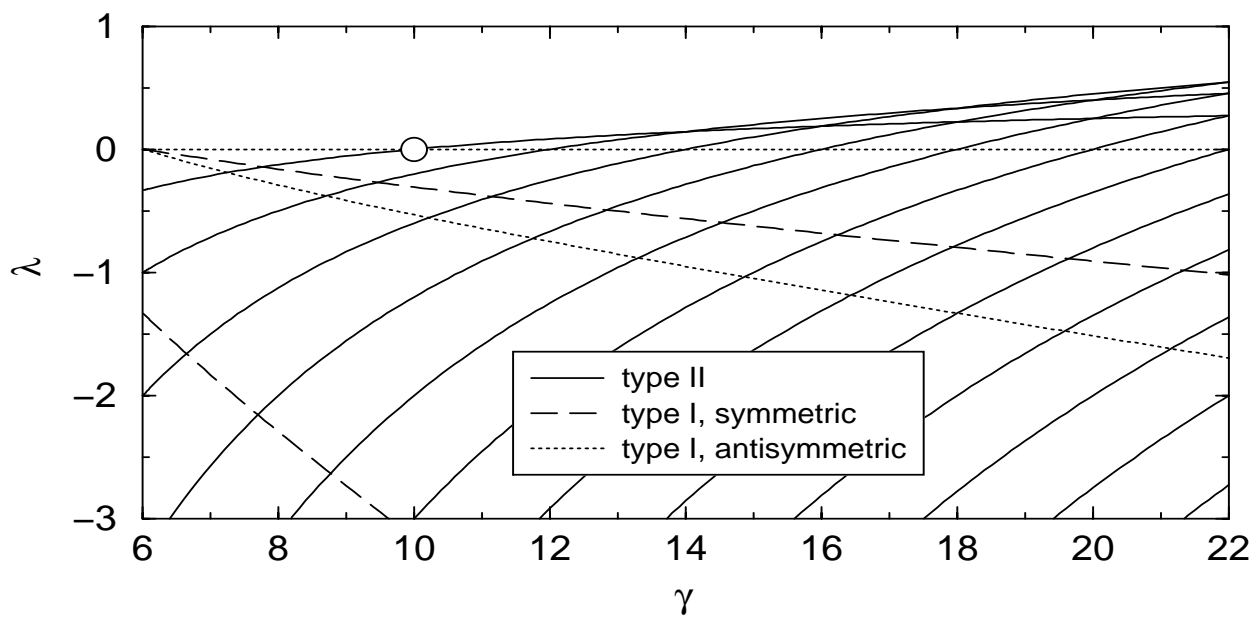

FIG. 2.8. Dependence of the eigenvalues $\lambda$ on $\gamma$ for the two-pole coalescent steady state $(N=2)$ which exists for $\gamma>6$. At $\gamma=10$ the largest eigenvalue crosses the horizontal axis (the point marked with an open circle) and the two-pole coalescent steady state becomes unstable. 
antisymmetric eigenvalues of type I is

$$
\lambda_{1}^{2, a}=0 ;
$$

the associated eigenfunction is given by (I-4.52) with $A_{1}=A_{2}$. The other antisymmetric eigenvalue is

$$
\lambda_{2}^{2, a}=-\tilde{\alpha}\left[\sinh ^{-2}\left(\frac{y_{1}-y_{2}}{2}\right)+\sinh ^{-2}\left(\frac{y_{1}+y_{2}}{2}\right)\right]=\sinh (\ln 6 \tilde{\alpha}) ;
$$

the associated eigenfunctions are given by (I-4.52) with $A_{1}=-A_{2}$. The two symmetric eigenvalues of type I are

$$
\begin{aligned}
\lambda^{2, s} & =-\tilde{\alpha} \frac{1}{2}\left\{\left[\sinh ^{-2}\left(\frac{y_{2}-y_{1}}{2}\right)+\sinh ^{-2}\left(\frac{y_{2}+y_{1}}{2}\right)\right]+\left[\sinh ^{-2} y_{1}+\sinh ^{-2} y_{2}\right]\right. \\
& \mp \sqrt{\left.\left[\sinh ^{-2}\left(\frac{y_{2}-y_{1}}{2}\right)-\sinh ^{-2}\left(\frac{y_{2}+y_{1}}{2}\right)\right]^{2}+\left[\sinh ^{-2} y_{1}-\sinh ^{-2} y_{2}\right]^{2}\right\}} ;
\end{aligned}
$$

the associated eigenfunctions are given by (I-4.51) with $A_{2}=\left(B \pm \sqrt{B^{2}+1}\right) A_{1}$, where

$$
B=\frac{\sinh ^{-2} y_{1}-\sinh ^{-2} y_{2}}{\sinh ^{-2}\left(\frac{y_{2}-y_{1}}{2}\right)-\sinh ^{-2}\left(\frac{y_{2}+y_{1}}{2}\right)} .
$$

The eigenvalues are plotted in Figure 2.8. The dependence of the eigenfunctions of type I of the two-pole solution on the parameter $\gamma$ is illustrated in Figures 2.9-2.12; they exhibit a behavior similar to that of the eigenfunctions of type I of the one-pole solution. The principal trend in the development of the eigenfunctions of type I, as $\gamma$ increases from $\gamma_{2}$ to $\infty$, is to become more singular.

There are infinitely many eigenvalues of type II given by formula (1.4), where $N=2$, and plotted in Figure 2.8. Each eigenvalue of type II has multiplicity two. Thus, each possesses two eigenfunctions - one symmetric and one antisymmetric. The dependence of the eigenfunctions of type II of the two-pole solution on the parameter $\gamma$ is illustrated in Figures 2.13-2.15. The principal trend in the development of the $m$ th eigenfunctions of type II $(m=3, \ldots, \infty)$, as $\gamma$ increases from $\gamma_{2}$ to $\infty$, is the transfer of energy, which has been initially (at $\gamma=\gamma_{2}$ ) fairly equally distributed over a wide range of wavenumbers, to the $(m-2)$ th wavenumber.

The character of the dependence of the eigenfunctions of the two-pole solution on the parameter $\gamma$ is prototypical for that of a general $N$-pole solution, with $N \geq 2$; the discussion on this dependence is presented in the next subsection.

2.4. $N$-pole solution. We first examine the dependence of the eigenfunctions of an $N$-pole coalescent steady state (with $N \geq 2$ ) on the parameter $\gamma$ (illustrated for the case $N=2$ in Figures 2.9-2.15) and then address the stability issues.

Eigenfunctions of type I (Figures 2.9-2.12). The principle trend in the development of any eigenfunction of type $\mathrm{I}$, as $\gamma$ increases from $\gamma_{N}$ to $\infty$, is to become more singular. The energy concentrated mostly near $k=1$, at the bifurcation point, $\gamma=\gamma_{N}$, is redistributed in such a way that the relative significance of the higher 

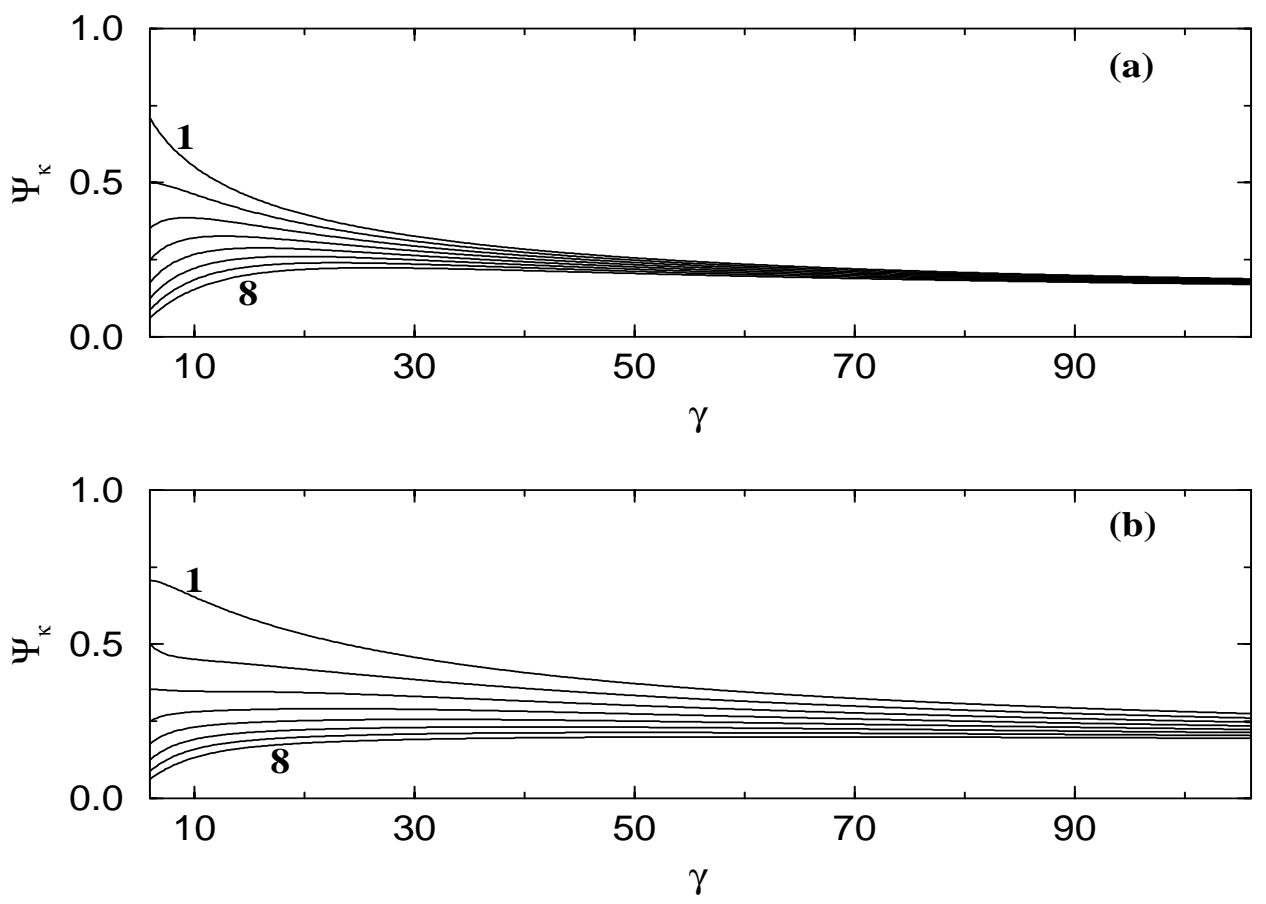

FIG. 2.9. Dependence of the first eight harmonics of the first symmetric (a) and antisymmetric (b) eigenfunctions (type I) of the two-pole coalescent steady state on $\gamma$.


FIG. 2.10. Dependence of the first eight harmonics of the second symmetric (a) and antisymmetric (b) eigenfunctions (type I) of the two-pole coalescent steady state on $\gamma$. 



FIG. 2.11. $k$-space representation (left column) and $x$-space representation (right column) of the first symmetric (row (a)) and antisymmetric (row (b)) eigenfunctions (type I) of the two-pole coalescent steady state at two values of parameter $\gamma$ : the lighter lines correspond to $\gamma=6.1$ and the darker lines correspond to $\gamma=100.1$.
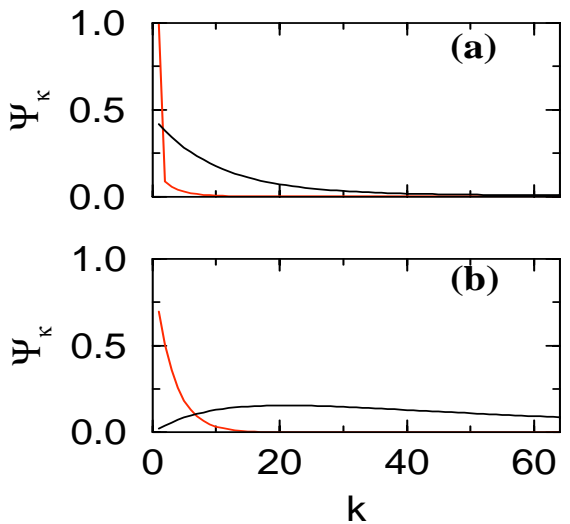
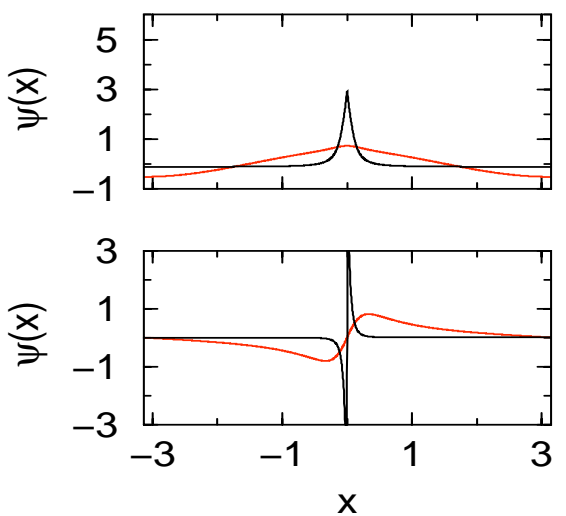

FIG. 2.12. $k$-space representation (left column) and $x$-space representation (right column) of the second symmetric (row (a)) and antisymmetric (row (b)) eigenfunctions (type I) of the two-pole coalescent steady state at two values of the parameter $\gamma$ : the lighter lines correspond to $\gamma=6.1$ and the darker lines correspond to $\gamma=100.1$.

harmonics increases at the expense of the lower ones. As $\gamma \rightarrow \infty$, the eigenfunction in $k$-space flattens out; in $x$-space a spike, or a pair of spikes, develops near the origin for the symmetric/antisymmetric eigenfunctions, respectively. The difference between any two eigenfunctions, for example, the $m$ th eigenfunction of an $M$-pole solution and the $n$th eigenfunction of an $N$-pole solution, is mostly quantitative.

Eigenfunctions of type II (Graphs (a)-(c) of Figures 2.13-2.14 and Figure 2.15; case $m=4$ is presented). The principle trend in the development of the mth eigenfunction of type II $(m=N+1, \ldots, \infty)$, as $\gamma$ increases from $\gamma_{N}$ to $\infty$, is the transfer of energy, which is first (at $\gamma=\gamma_{N}$ ) fairly equally distributed over a wide range of wavenumbers, to the $(m-N)$ th wavenumber. This transfer of energy does not occur directly but in two stages, which are similar to the intermediate and last stages in the development of the type II eigenfunctions of the one-pole solution. At the beginning 

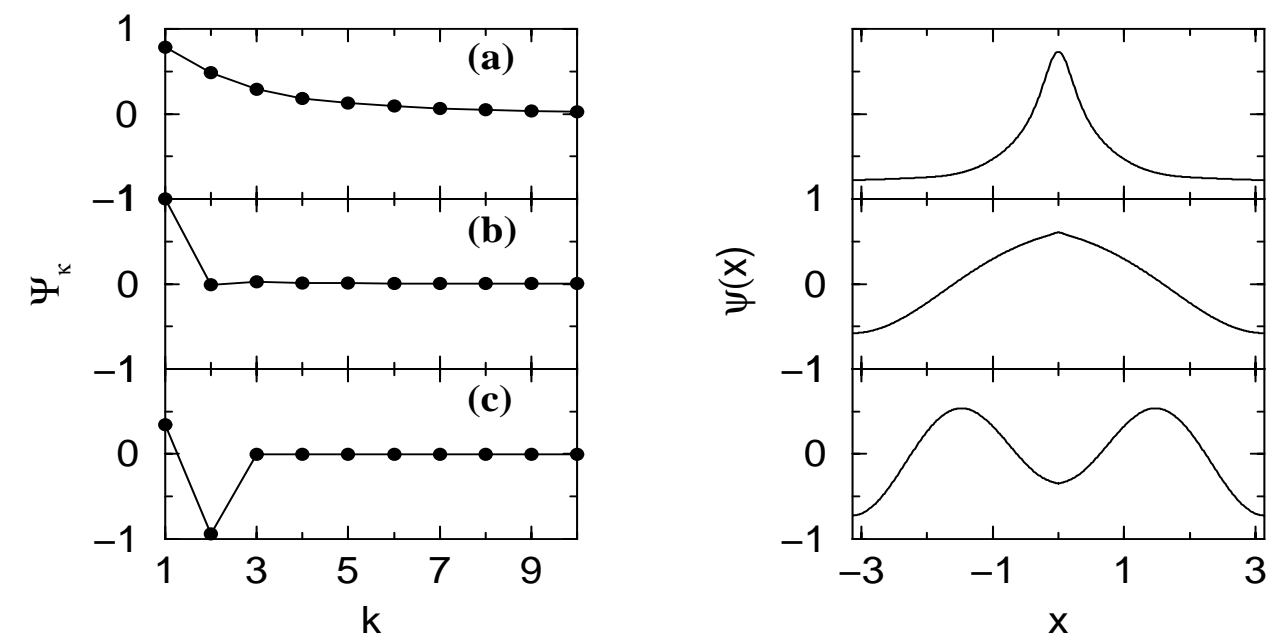

FIG. 2.13. $k$-space representation (left column) and $x$-space representation (right column) of the fourth symmetric eigenfunction (type II) of the two-pole coalescent steady state at different values of the parameter $\gamma$ : (a) $\gamma=6.1$, (b) $\gamma=16$, and (c) $\gamma=100.1$.
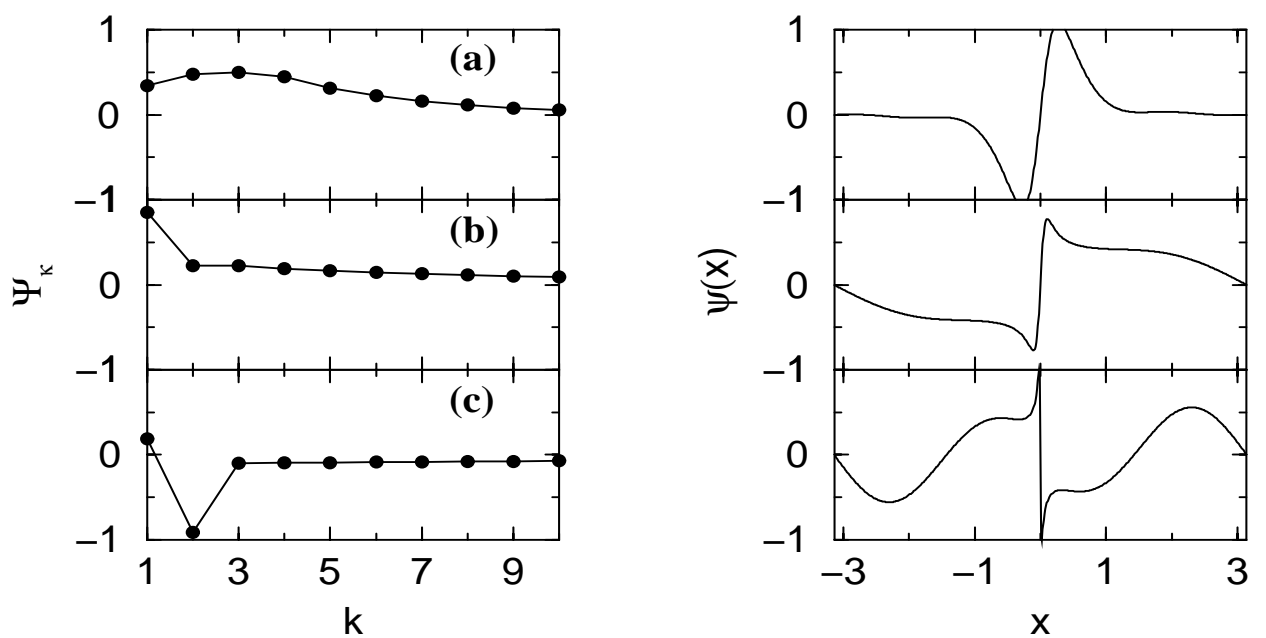

FIG. 2.14. $k$-space representation (left column) and $x$-space representation (right column) of the fourth antisymmetric eigenfunction (type II) of the two-pole coalescent steady state at different values of the parameter $\gamma$ : (a) $\gamma=6.1$, (b) $\gamma=18$, and (c) $\gamma=100.1$.

of the first stage, at $\gamma=\gamma_{N}$, the symmetric eigenfunction $\psi_{m}^{N, s}$ bifurcates from $\psi_{m}^{N-1, s}$ which has a bell shaped profile and the antisymmetric eigenfunction $\psi_{m}^{N, a}$ bifurcates from $\psi_{m}^{N-1, a}$ which has a "double bell"-shaped profile; in both cases the energy is fairly equally distributed over a wide range of wavenumbers (a). As $\gamma$ increases during the first stage most of the energy is first transferred to the first wavenumber (b), and then from the first to the second wavenumber, from the second wavenumber to the third wavenumber, etc., ending up in the $(m-N)$ th wavenumber. At the end of this stage, the $k$-space representation is dominated by the maximum (if $m-N$ is odd) 

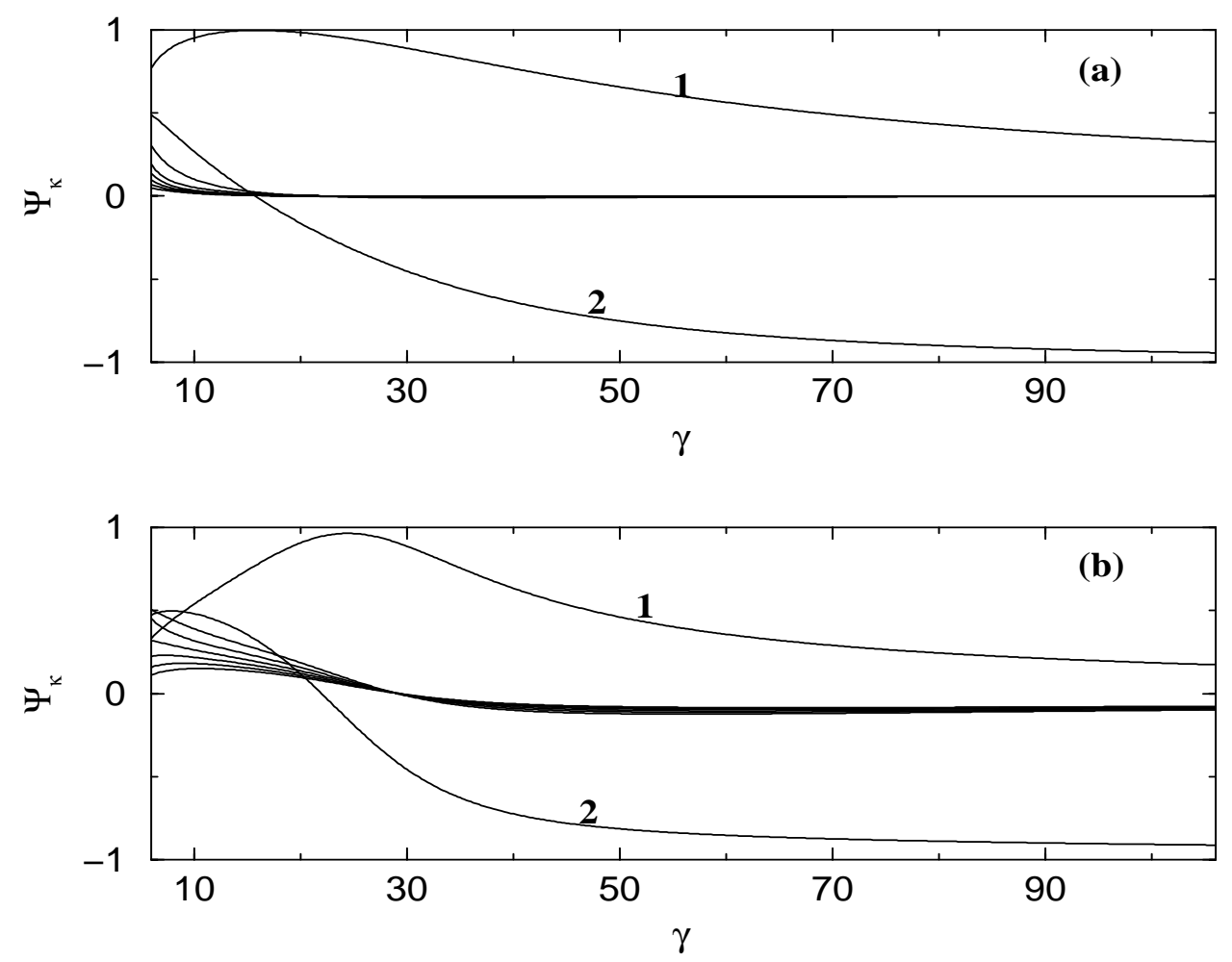

FIG. 2.15. Dependence of the first eight harmonics of the fourth symmetric (a) and antisymmetric (b) eigenfunctions (type II) of the two-pole coalescent steady state on $\gamma$.

or minimum (if $m-N$ is even) at $k=m-N$; though some small contributions from all the remaining wavenumbers are still recognizable. In $x$-space, although the $m$ th eigenfunction is close to a cosine or sine with wavenumber $(m-N)$, a sharp change occurs near the origin; the singular behavior there is quite obvious in the antisymmetric case (see Figure 2.14(b), right column) but can be also detected in the symmetric case (see Figure 2.13(b), right column). In the second stage, when values of $\gamma$ are large, the $(m-N)$ th wavenumber drains the energy from the rest of the wavenumbers; the energy contained in the $(m-N)$ th wavenumber increases asymptotically approaching 1 while the energy of all other wavenumbers decrease asymptotically approaching 0 (c). As $\gamma \rightarrow \infty$ the shape of an antisymmetric eigenfunction in $x$-space approaches a pure sine harmonic with the wavenumber $m-N$ and the shape of a symmetric eigenfunction in $x$-space approaches a pure cosine harmonic with the wavenumber $m-N$. The singular region near the origin shrinks and disappears in the limit.

We point out that the energy of either a symmetric or an antisymmetric eigenfunction that corresponds to $k=1, \ldots, m-N-1$ decays like $\gamma^{-2}$. However, the energy of a symmetric eigenfunction that corresponds to $k=m-N+1, \ldots, \infty$, decays like $\gamma^{-6}$, while that of an antisymmetric eigenfunction decays like $\gamma^{-2}$. For this reason, the singularity in the figures near $x=0$ is more obvious in the antisymmetric case than in the symmetric case.

The linear stability results for an $N$-pole solution $\varphi_{N}(x)(N \geq 1)$ follow immediately from (1.4) and the fact that all eigenvalues of type I are nonpositive. These imply 
that there exists a critical value of the parameter $\gamma=1 / \tilde{\alpha}$, namely $\gamma_{N+1}=2(2 N+1)$ such that

(1) for $\gamma_{N}<\gamma<\gamma_{N+1}$ all eigenvalues are nonpositive, the solution $\varphi_{N}(x)$ is neutrally stable;

(2) the $(N+1)$ th eigenvalue, $\lambda_{N+1}^{N}$, becomes zero at $\gamma=\gamma_{N+1}$, at this point the solution $\varphi_{N}(x)$ is marginally stable;

(3) for $\gamma>\gamma_{N+1}$ the $(N+1)$ th eigenvalue, $\lambda_{N+1}^{N}$, is positive, the solution $\varphi_{N}(x)$ is unstable.

By neutrally stable, we mean that, apart from the trivial translational mode, the solution is asymptotically stable: There is only one eigenfunction (the translational mode) associated with a zero eigenvalue; all other eigenfunctions are stable, corresponding to negative eigenvalues. The translational mode, related to a lateral shift of the flame front with no change in the shape of the front, is of little interest from the practical point of view. By marginally stable we mean that, apart from the trivial translational mode, there is at least one other nontrivial eigenfunction corresponding to the zero eigenvalue. Here, there are two such eigenfunctions, the symmetric and the antisymmetric ones, both associated with the same eigenvalue $\lambda_{N+1}^{N}$. The eigenspace of the zero eigenvalue is, therefore, three-dimensional. (Things are different with the flat flame solution, $N=0$, which does not possess a zero eigenvalue with the translational mode. When this solution becomes marginally stable, the eigenspace of a zero eigenvalue is two-dimensional.)

3. Poles and eigenvalues; cascading bifurcations. Based on the preceding results, a clear pattern develops regarding the existence and linear stability of the family of coalescent steady states. Suppose we carry out an experiment in which $\gamma$, the only parameter of the problem, increases from 0 to $\infty$, and observe the changes in the existence and stability properties of the solutions. As it turns out qualitative changes occur at the points $\gamma=\gamma_{N+1}, N=0, \ldots, \infty$, so that it becomes convenient to focus our attention on the intervals $I_{N}=\left(\gamma_{N} ; \gamma_{N+1}\right)$ and in particular on the transition that takes place as we move from one interval to the adjacent one. As in Part I, we assumed $\gamma_{0}=0$.

We begin with the first interval, $I_{0}$. Only one solution from the family of coalescent steady states exists in this interval; it is the zero-pole state $\varphi_{0}$. This solution has an infinitely countable number of poles and an infinitely countable number of eigenvalues. All the poles are of type II; i.e., they are at infinity. All the eigenvalues are of type II: they are of multiplicity two and, within the interval $I_{0}$, they are all negative. The zero-pole state is asymptotically stable in the interval $I_{0}$. Upon reaching the end of the interval $I_{0}$, i.e., at $\gamma=\gamma_{1}$, the largest eigenvalue, $\lambda_{1}^{0}$, becomes zero; $\varphi_{0}$ becomes marginally stable (see Figure 2.1 ). However, at this point the one-pole state $\varphi_{1}$ bifurcates from the zero-pole state $\varphi_{0}$.

Consider now a general interval, $I_{N-1}$. There exist $N$ solutions of the family of coalescent steady states in this interval. The first $N-1$ solutions, $\varphi_{0}, \varphi_{1}, \ldots, \varphi_{N-2}$, are clearly unstable. We thus focus on the last solution, the $(N-1)$-pole solution $\varphi_{N-1}$. This solution has an infinitely countable number of poles and an infinitely countable number of eigenvalues. Among the poles $N-1$ are of type I, i.e., they are at a finite distance from the real axis, and the rest are of type II, i.e., they are at infinity. Among the eigenvalues $N-1$ are symmetric of type I and $N-1$ are antisymmetric of type I; the rest are of type II. Each eigenvalue of type I is simple. Each eigenvalue of type II has multiplicity two. Within the interval $I_{N-1}$ all the eigenvalues are negative. The $N$-pole state is asymptotically stable in the interval $I_{N-1}$. Upon reaching the 

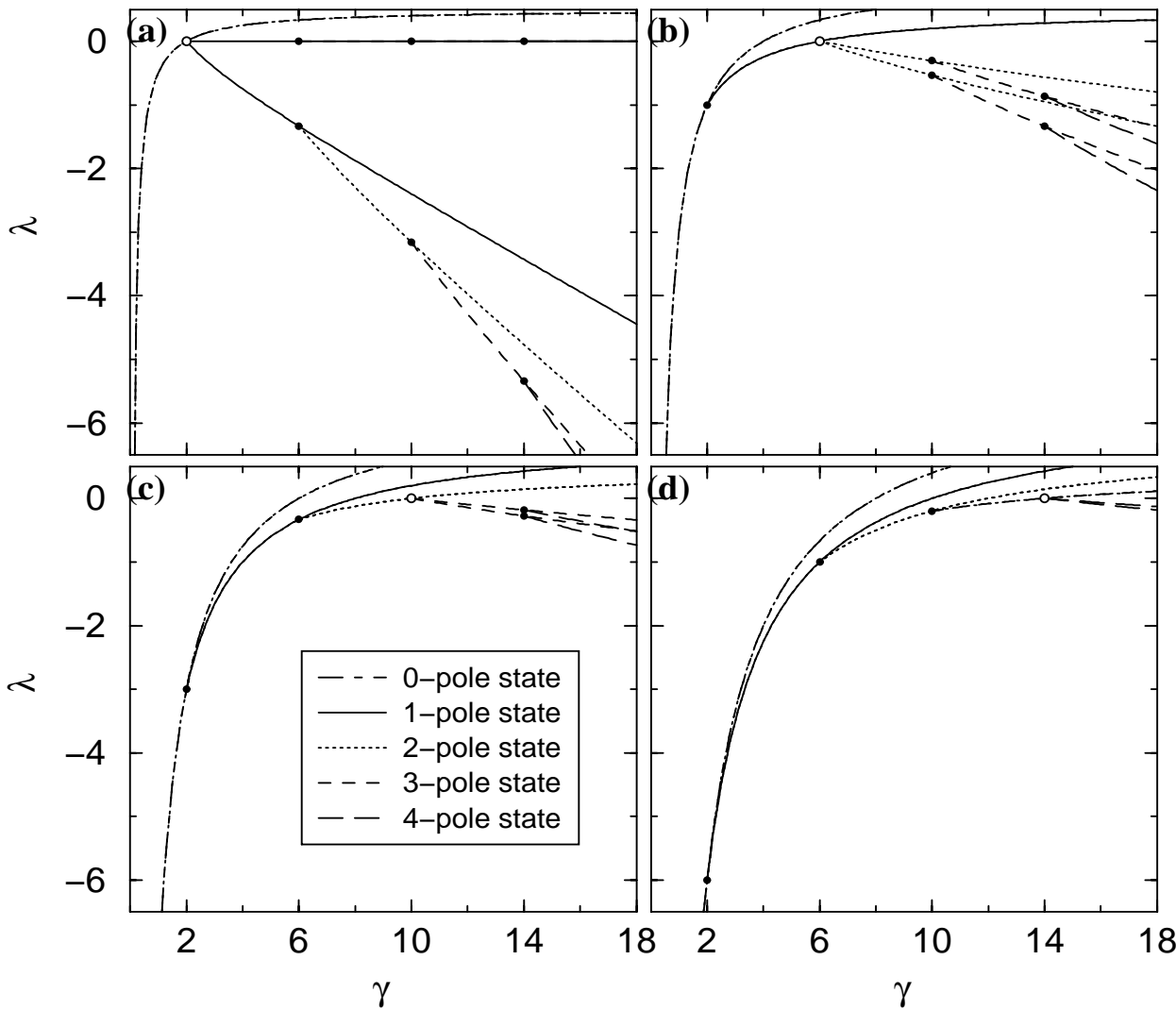

FIG. 3.1. Dependence of the first (a), second (b), third (c), and fourth (d) eigenvalues of the $N$ pole coalescent steady states, $N=0, \ldots, 4$, on $\gamma$. The points where the eigenvalues of the newborn states bifurcate from the eigenvalues of the parent states are marked with circles. When both the parent and the newborn eigenvalue belong to the same type a filled circle is used. When the parent eigenvalue is of type II but the newborn eigenvalue is of type I, an open circle is used.

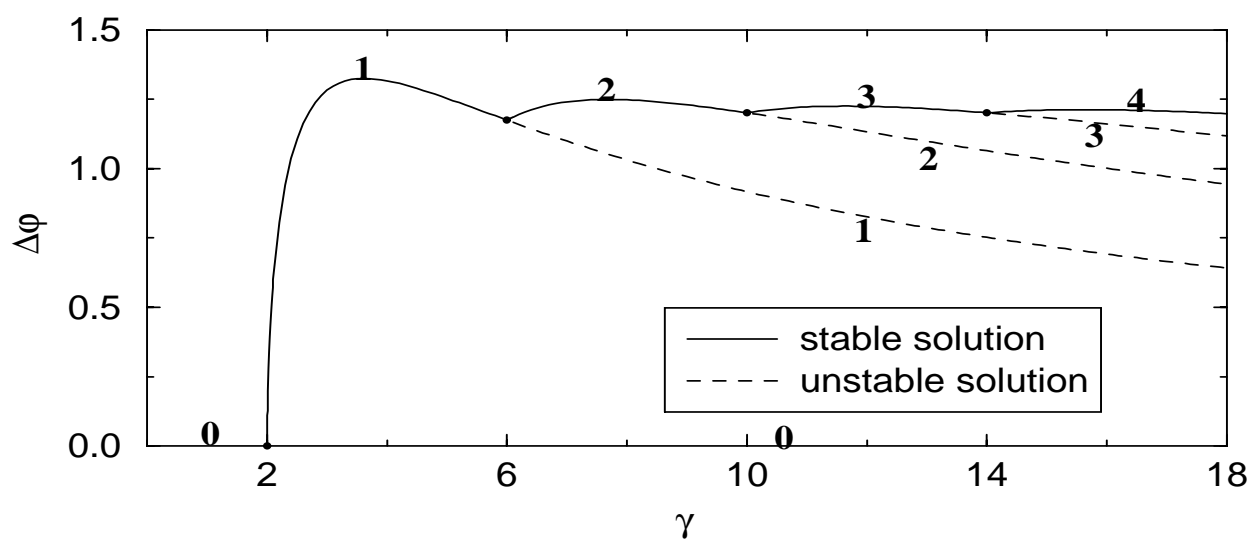

FIG. 3.2. Dependence of the amplitude, $\Delta \varphi$, of the $N$-pole coalescent steady states, $N=$ $0, \ldots, 4$, on $\gamma$. The points where the new states are born are marked with dots. 
end of the interval $I_{N-1}$, i.e., at $\gamma=\gamma_{N}$ the largest eigenvalue $\lambda_{N}^{N-1}$ becomes zero; $\varphi_{N-1}$ becomes marginally stable (see, for example, Figures 2.2 and 2.8). However, at this point the $N$-pole state $\varphi_{N}$ bifurcates from the $(N-1)$-pole state $\varphi_{N-1}$. The bifurcation at $\gamma=\gamma_{N}$ is characterized by the following two properties:

(1) All the poles of $\varphi_{N}$ bifurcate from the poles of $\varphi_{N-1}$ (see Figure 1.1). The $N$ th pole of $\varphi_{N}$ is of the opposite type from its parent pole. The rest of the poles of $\varphi_{N}$ inherent the types of their parent poles.

(2) All the eigenvalues of $\varphi_{N}$ bifurcate from the eigenvalues of $\varphi_{N-1}$ (see Figure 3.1). In particular:

(i) two simple eigenvalues of $\varphi_{N}, \lambda_{N}^{N, s}$ and $\lambda_{N}^{N, a}$, are born from one double zero eigenvalue of $\varphi_{N-1}, \lambda_{N}^{N-1}$;

(ii) the newborn eigenvalues $\lambda_{N}^{N, s}$ and $\lambda_{N}^{N, a}$ are of type I and, therefore, of the opposite type from their parent eigenvalue, $\lambda_{N}^{N-1}$, which is of type II;

(iii) the rest of the eigenvalues of $\varphi_{N}$ inherent the types of their parent eigenvalues, namely, $\lambda_{n}^{N, s}$ and $\lambda_{n}^{N, a}$, as well as $\lambda_{n}^{N-1, s}$ and $\lambda_{n}^{N-1, a}$, with $n=1, \ldots, N-1$, are of type I and $\lambda_{n}^{N}$, as well as $\lambda_{n}^{N-1}$, with $n=N+1, \ldots, \infty$, are of type II.

Immediately beyond the interval $I_{N-1}$, the largest eigenvalue of the $(N-1)$-pole state $\lambda_{N}^{N-1}$ is positive so that $\varphi_{N-1}$ is unstable. However, for the newborn $N$-pole state, the two eigenvalues that bifurcated from $\lambda_{N}^{N-1}, \lambda_{N}^{N, s}$, and $\lambda_{N}^{N, a}$ are negative. All the other eigenvalues of $\varphi_{N}$, like those of $\varphi_{N-1}$, are negative with the exception of $\lambda_{1}^{N, a} \equiv \lambda_{1}^{N-1, a} \equiv 0$. Thus, apart from a translational mode associated with $\lambda_{1}^{N, a}$, the solution $\varphi_{N}$ is stable. There is a supercritical bifurcation at the point $\gamma_{N}$ illustrated in Figure 3.2.

From the general pattern just described, it appears that there is a close relation between the poles and the eigenvalues of the $N$-pole coalescent steady state $\varphi_{N}$. The (linearized) dynamic of the $N$ poles of type I, located at a finite distance from the real axis, is linked to the $N$ symmetric and $N$ antisymmetric eigenvalues of type I and the associated eigenfunctions. The (linearized) dynamic of the " $\infty-N$ " poles of type II, located at infinity, is linked to the " $\infty-N$ " eigenvalues of type II and the associated eigenfunctions. At the bifurcation point a pole of type II moves from infinity to a finite location, becoming a pole of type I for the newborn solution. Similarly, an eigenvalue (with multiplicity two) of type II with a tendency of becoming positive splits into two simple eigenvalues of type I which are necessarily negative. In this way the newborn solution gains stability while its parent becomes unstable.

An important conclusion from the above results is that for any value of the parameter $\gamma$ there always exists one and only one nonnegative integer $N$ such that the $N$-pole solution from the family of coalescent steady states exists and is (apart from a translational mode) stable. It should be pointed out that this $N$ is the largest possible $N$ for this particular value of $\gamma$, i.e., $N_{0}(\gamma)$ defined in (I-3.24).

Next we would like to comment on the energy transfer that takes place at the bifurcations points as $\gamma$ increases from 0 to $\infty$. Recall that at $\gamma=\gamma_{N}$, an $m$ th eigenfunction of the $N$-pole solution $\psi_{m}^{N}$ bifurcates from an $m$ th eigenfunction of the $(N-1)$-pole solution $\psi_{m}^{N-1}$, where $N, m=1, \ldots, \infty$. For each eigenfunction the energy, defined at the beginning of section 2 , is a function of $\gamma$; we shall refer to its value as $\gamma \rightarrow \infty$ (i.e., when diffusion becomes negligibly small) as the limiting energy. There are three qualitatively different types of bifurcations from $\psi_{m}^{N-1}$ to $\psi_{m}^{N}$ that occur as long as the distribution of the limiting energy over the wavenumbers is concerned.

(i) When $m=1, \ldots, N-1$ either eigenfunction is of type I and the limiting 
energy is equally distributed over all wavenumbers.

(ii) When $m=N$, the parent eigenfunction $\psi_{N}^{N-1}$ is of type II with its limiting energy accumulated in the first, i.e., the lowest, wavenumber. The newborn eigenfunction $\psi_{N}^{N}$, however, is of type I with its limiting energy equally distributed over all wavenumbers.

(iii) When $m=N+1, \ldots, \infty$ either eigenfunction is of type II. However, while the limiting energy of the parent eigenfunction $\psi_{m}^{N-1}$ is accumulated in the $(m-N+$ 1)th wavenumber, the limiting energy of the newborn eigenfunction $\psi_{m}^{N}$ is accumulated in the $(m-N)$ th wavenumber.

Consider now the changes that occur when an $m$ th eigenfunction undergoes a cascade of bifurcations. The limiting energy is first accumulated in one mode. At each bifurcation the energy remains accumulated but is transferred to a lower mode; when this is no longer possible it gets redistributed over all modes, and remains so in all subsequent bifurcations. More precisely, all the limiting energy of $\psi_{m}^{0}$ is contained in the $m$ th wavenumber. First, at each bifurcation the limiting energy keeps on shifting one wavenumber down: at $\gamma=\gamma_{1}$ all the limiting energy of $\psi_{m}^{1}$ is contained in the $(m-1)$ th wavenumber, at $\gamma=\gamma_{m-1}$ all the limiting energy of $\psi_{m}^{m-1}$ is contained in the first wavenumber. At the next bifurcation $\gamma=\gamma_{m}$ the limiting energy can no longer be moved down, instead it gets redistributed over all wavenumbers. The limiting energy of $\psi_{m}^{n}$, with $n \geq m$, is equally distributed over all wavenumbers. After infinitely many bifurcations, i.e., when $N \rightarrow \infty(\gamma \rightarrow \infty)$, all eigenfunctions have their energy equally distributed over all wavenumbers; in $x$-space the shape of each eigenfunction is singular, it is a single spike (for symmetric eigenfunctions) and double spikes (for antisymmetric eigenfunctions) as described in section 2.

Consider now a "composite" solution of the PDE which, in terms of $\gamma$, is composed of those portions of the solutions that are stable. That is,

$$
\varphi_{C}(x ; \gamma)=\varphi_{N}(x), \quad \text { if } \gamma \in I_{N}, \quad N=0, \ldots, \infty .
$$

We note parenthetically that this "solution" has been examined in [6, 7]. It is obvious that apart from a trivial translational mode, this solution is asymptotically stable for all values of $\gamma$.

The dependence of the reciprocals of the imaginary parts of the poles of the "composite" solution is shown in Figure 3.3. The number of the finite poles of the "composite" solution jumps from $N-1$ to $N$ at the point $\gamma_{N}, N=1, \ldots, \infty$. To preserve stability, at the point where the solution $\varphi_{N-1}$ is about to become unstable, the PDE switches to the stable solution $\varphi_{N}$. In other words, a new pole comes down from infinity, changes the structure of the solution, and ensures its stability.

The spectrum corresponding to the "composite" solution is shown in Figure 3.4. This figure illustrates the peculiar dependence of a typical eigenvalue of the "composite" solution, $\lambda_{N}$, on $\gamma$. (To help visualization, the dependence of $\lambda_{6}$ on $\gamma$ is singled out with bold lines.) A typical eigenvalue $\lambda_{N}(N>1)$ increases piecewise smoothly from $-\infty$ to 0 as $\gamma$ increases in the interval $\left(\gamma_{0}, \gamma_{N}\right)$. $\lambda_{N}$ has multiplicity two on this interval; there are two eigenfunctions, one symmetric and one antisymmetric, associated with it. At the point $\gamma_{N}$ the eigenvalue hits the horizontal axis and then bounces back splitting into two simple eigenvalues $\lambda_{N}^{s}$ and $\lambda_{N}^{a}$, one corresponding to the symmetric eigenfunction and the other to the antisymmetric eigenfunction. The eigenvalues $\lambda_{N}^{s}$ and $\lambda_{N}^{a}$ then decrease piecewise smoothly from 0 to $-\infty$ as $\gamma$ keeps on increasing on the interval $\left(\gamma_{N}, \infty\right)$. It is always true that $\lambda_{N}^{s}>\lambda_{N}^{a}$ on this interval. The first eigenvalue, $\lambda_{1}$, exhibits qualitatively the same dependence on the parameter $\gamma$ with the only exception that $\lambda_{1}^{a}$ stays identical zero on the interval $\left(\gamma_{1}, \infty\right)$. 


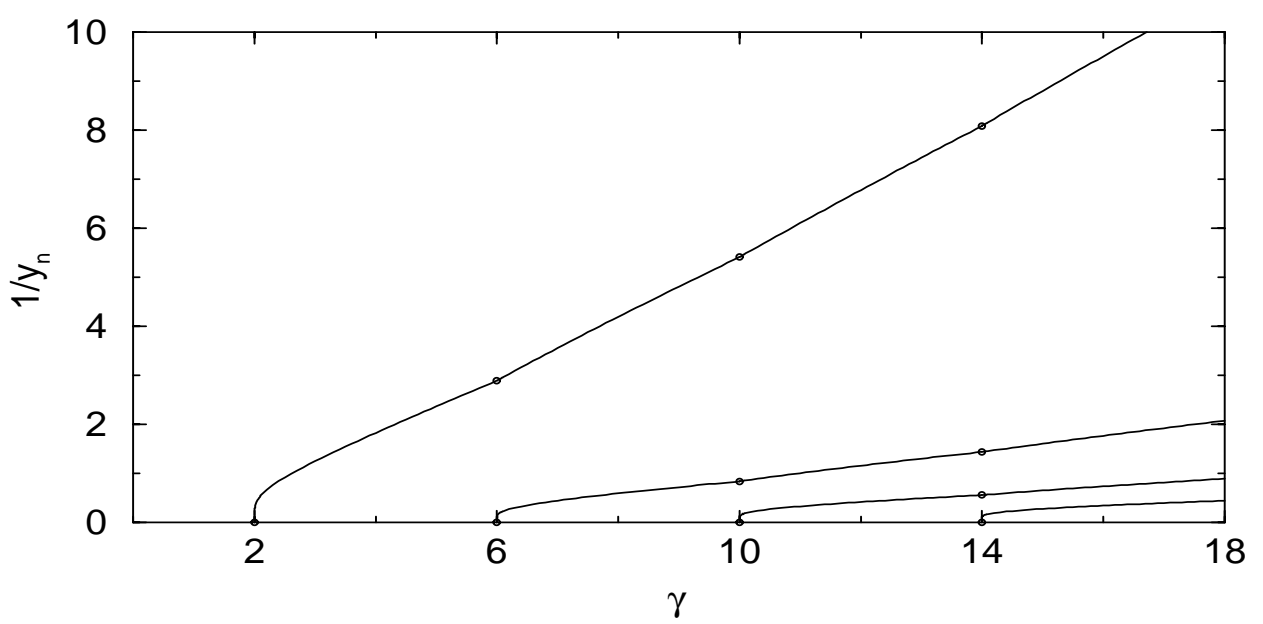

FIG. 3.3. Dependence of the reciprocals of the imaginary parts of poles, $1 / y_{n}$, on $\gamma$ for the "composite" solution. Dots indicate the points where the "composite" solution switches from the $N$-pole coalescent steady state to the $(N+1)$ th coalescent steady state.

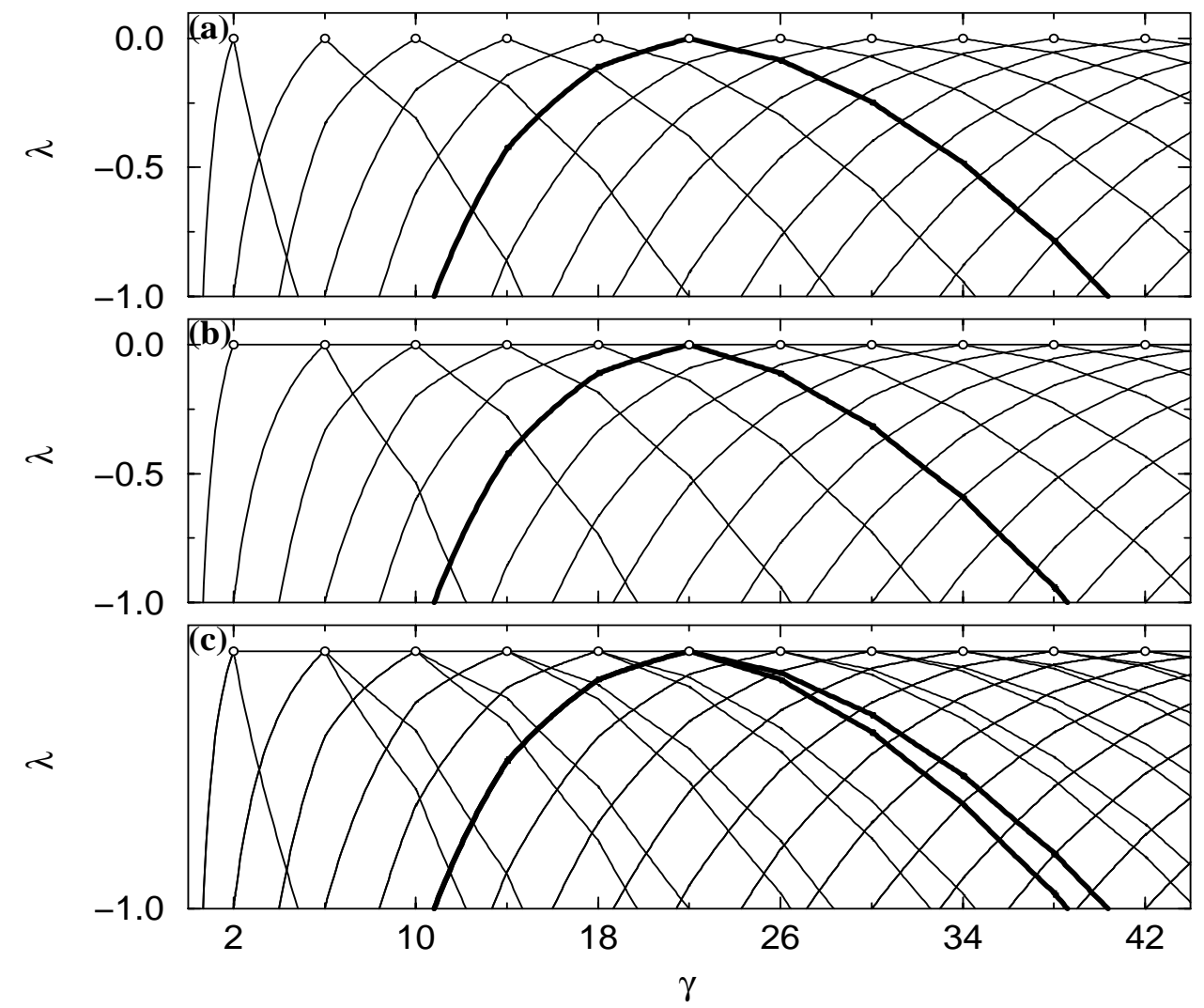

FIG. 3.4. Dependence of the symmetric (a), antisymmetric (b), and all (c) eigenvalues $\lambda$ on $\gamma$ for the "composite" solution. Open circles indicate the points at which the eigenvalues change their type from II to I. Bold lines single out the sixth eigenvalue, $\lambda_{6}$. 
The dependence of the amplitude $\Delta \varphi$, as defined in Part I, of the "composite" solution on $\gamma$ is shown in Figure 3.2 with a solid line. It should be pointed out that the amplitude tends to a constant value as $\gamma \rightarrow \infty$. It is just a consequence of the fact that the shape of the coalescent steady state tends to a genuine cusp as $\gamma \rightarrow \infty$ : the limiting value of the amplitude of the "composite" solution is the amplitude of that cusp.

4. Summary and discussion. Previous studies addressing the linear stability of steady coalescent pole solutions were reported in $[5,6,7,8]$. In $[6,7]$ the infinite linear system (I-4.6) was truncated at some cutoff value $k^{*}$ and the eigenvalues of the truncated matrix were sought numerically. The results are summarized in [7, Figure 1] which shows the spectrum of what is referred to in the present work as the composite solution (3.1). All eigenvalues appear negative with the exception of some positive eigenvalues for $\gamma>\gamma_{9}$. Without commenting on these seemingly unstable modes the authors concluded that the $N$-pole coalescent steady state is neutrally stable for all positive integral values of $N$. Their results also imply that the eigenspace associated with the zero eigenvalue is one-dimensional for all values of $\gamma$ in the open intervals $I_{N}$, $N>0$, but is three-dimensional at the end points of these intervals, i.e., at $\gamma=\gamma_{N+1}$. In $[5,8]$ the linear PDE (I-4.3) for the flame front displacement perturbation $\psi(x, t)$ was integrated numerically. It was reported that, regardless of the initial conditions, an asymptotic state of the form $\psi(x, t)=e^{\tilde{\lambda} t} \Psi(x)$ was always reached, with $\tilde{\lambda}$ a real number. When $\tilde{\lambda}$ was positive the steady state was considered unstable with $\Psi(x)$ corresponding to the most linearly unstable mode and $\tilde{\lambda}$ its growth rate. The steady state was considered stable when $\tilde{\lambda}$ was negative and neutrally stable when $\tilde{\lambda}$ was zero. As reported by the authors in [5] their results contradict those of $[6,7]$ listed above in two ways: (i) The $N$-pole coalescent steady state is neutrally stable in the interval $I_{N}$ for $N=1,2,3$, but is unstable for $N \geq 4$ for all values of $\gamma$. (ii) For those values of $\gamma$ for which the $N$-pole coalescent steady state is reported to be neutrally stable, the eigenspace associated with the zero eigenvalue, $\tilde{\lambda}=0$, is (at least) two-dimensional.

The conclusions of $[6,7]$ agree, in principle, with the results of our exact solutions except for inaccuracies and interpretation. The conclusions drawn in $[5,8]$, on the other hand, do not agree with our exact results; we have proved unequivocally that the $N$-pole coalescent steady states are neutrally stable in the intervals $I_{N}$ for all positive integral values of $N$. We believe that the erroneous positive growth rates $\tilde{\lambda}$ reported in [8] for $N \geq 4$ result from the relatively low number of modes included in their calculations for the relatively large values of $\gamma$ associated with these cases. This will be further discussed below. Furthermore, it is straightforward to conclude from our results that the eigenspace associated with the zero eigenvalue of the $N$-pole coalescent steady state $(N \geq 1)$ is one-dimensional for all values of $\gamma$ in the open interval $I_{N}$ with the exception of the end point of the interval, $\gamma_{N+1}$. At this point the largest eigenvalue of type II (with multiplicity two) crosses the horizontal axis so that the eigenspace associated with the zero eigenvalue is three-dimensional.

After properly rescaling the variables, [7, Figure 1] agrees with our Figure 3.4 (c) except for the positive eigenvalues appearing for $\gamma>\gamma_{9}$. It is clear from the present work that these growing modes could result from an accumulated error originating from a cutoff value $k^{*}$ that is too small. The choice $k^{*}=4 k_{\max }$ was adopted in [7], with $k_{\max }=\gamma / 2$ the largest wavenumber $k$ for which the flat front solution is linearly unstable. Although this number is large enough to compute the spectrum of a flat front $(N=0)$ for any value of $\gamma$, it is not sufficiently large for accurately computing the spectrum of an $N$-pole solution, with $N>0$, for large enough values of $\gamma$. We 
recall that the eigenfunctions of the zero-pole solution are the only ones that do not depend on $\gamma$; for all the other solutions the eigenfunctions (especially of type I) in Fourier space flatten out as $\gamma$ or $N$ increases. Thus, the number of modes needed to represent such eigenfunctions adequately increases drastically with $\gamma$ and/or $N$. It should be pointed out that, although the results in [7] for relatively small values of $\gamma$ are sufficiently accurate, the interpretation of the data as presented in their Figure 1 is not correct. The interpolation between the numerically computed points in [7, Figure 1], which was purely speculative, led the authors to conclude that the eigenvalues of the "composite" solution oscillate periodically with a dependence on $L$ like $L^{-2}$. Note that in their figure $\tilde{\lambda} L^{2}$ was plotted against $L$, where $L \sim \gamma$ is the size of the domain of integration. It is clear from our exact representation of eigenvalues shown in Figure 3.4 that the eigenvalues of the composite solution do not oscillate, but rather increase monotonically from $-\infty$, hit the horizontal axis and bounce back decreasing monotonically to $-\infty$. Indeed, by connecting points associated with different eigenvalues one may be led to the erroneous conclusion that the eigenvalues oscillate. Furthermore, as can be easily seen from the formulas (I-4.24) and (I-4.37), the dependence of $\tilde{\lambda}$ on $L$ is somewhat more complex than just being $\sim L^{-2}$.

It is instructive to elaborate on the special cases discussed in $[5,8]$. In [5], the linear stability of the first three steady coalescent pole solutions was examined. The cases studied were (a) one-pole solution $(N=1)$ in the interval $L=6 \pi$, corresponding to $\gamma=3$; (b) two-pole solution $(N=2)$ in the interval $L=14 \pi$, corresponding to $\gamma=7$; and (c) three-pole solution $(N=3)$ in the interval $L=23 \pi$, corresponding to $\gamma=23 / 2$. In each of the three cases the calculated growth rate $\tilde{\lambda}$ was zero, implying that the steady state is neutrally stable. In each of the three cases only one nontrivial eigenmode was found; these were plotted in their Figure 7 . The authors also pointed out that the shape of the eigenmodes in all three cases was strikingly similar. As the number of poles in the solution increased, the maximum and minimum of eigenmodes got closer to the boundaries of the interval. Since the values of $\gamma$ in the three cases considered belong to the intervals $I_{1}, I_{2}$, and $I_{3}$, respectively, our results show that the one-, two-, and three-pole solutions are indeed neutrally stable. The largest eigenvalue in each of the three cases (the first two cases are shown in Figures 2.2 and 2.8) is the first antisymmetric eigenvalue $\lambda_{1}^{N, a} \equiv 0(N=1,2,3)$ which is a simple eigenvalue (being of type I). Each eigenvalue possesses only one antisymmetric eigenfunction which corresponds to the translational mode $\psi_{1}^{N, a}(x)=$ const $\frac{d}{d x} \varphi_{N}(x)$. The steady states $\varphi_{N}(x)$ are all similar in shape except for the depth of their cusp; the larger $N$, the deeper the cusp or the more "singular" is the solution at $x=0$. It is therefore not surprising that the shapes of all three eigenmodes are similar. As the number of poles increases the extrema of the eigenmodes move towards the boundaries of the interval and become sharper; see Figures 4.1 (a)-(c). We note that, although in this paper we work with the domain $(-L, L)$, the eigenfunctions in Figures 4.1 and 4.2 are presented on the interval $(0,2 L)$ for easy comparison with $[5,8]$. There is a complete agreement between the numerical calculations of [5] and our exact solutions in these cases.

In [8], the linear stability of the three steady coalescent pole solutions with $N=$ $6,8,10$ was examined. The cases studied were (d) six-pole solution $(N=6)$ in the interval $L=52 \pi$, corresponding to $\gamma=26$; (e) eight-pole solution $(N=8)$ in the interval $L=68 \pi$, corresponding to $\gamma=34$; and (f) ten-pole solution $(N=10)$ in the interval $L=82 \pi$, corresponding to $\gamma=41$. In each of these three cases the solution 

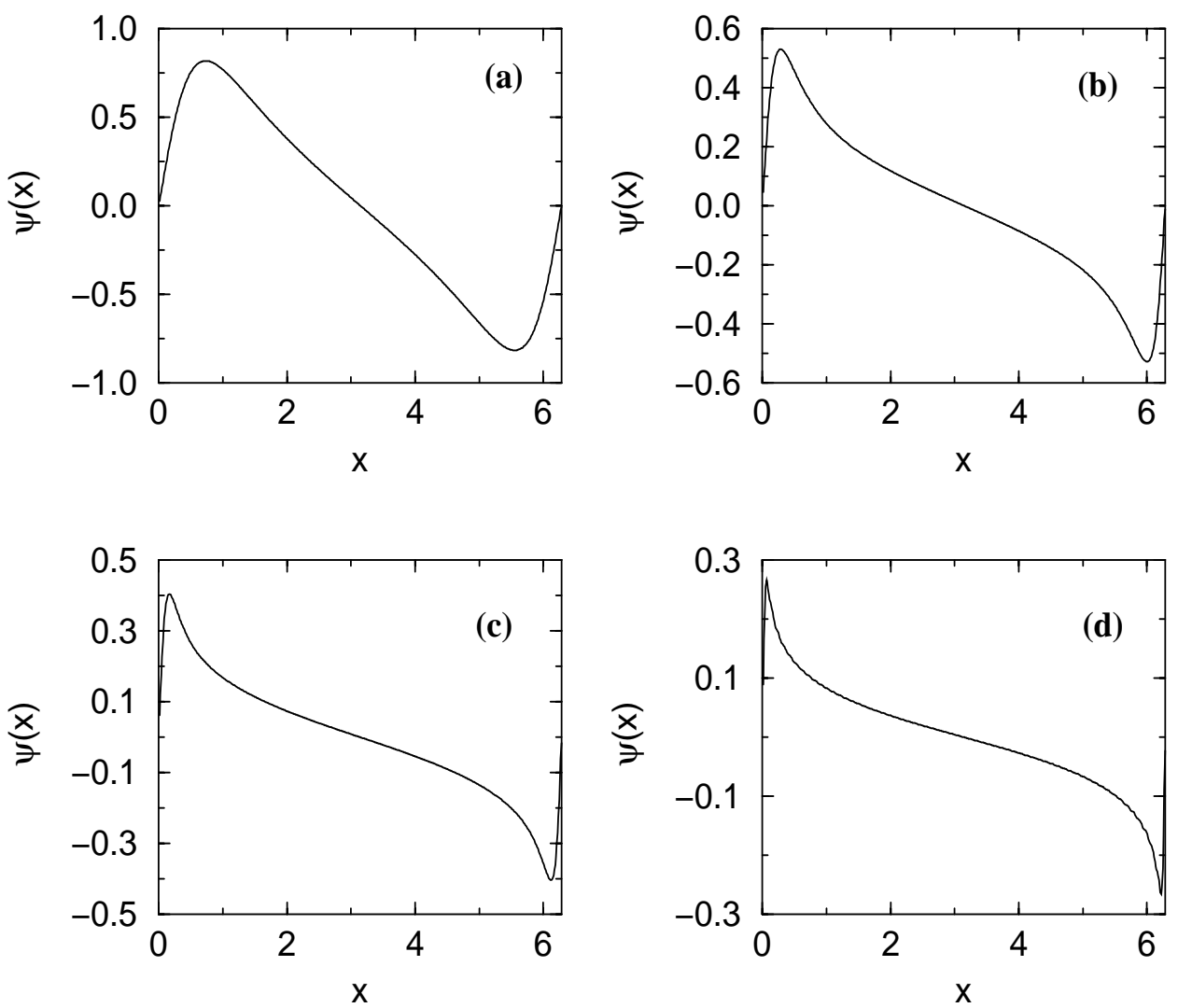

FIG. 4.1. $x$-space representation of the first antisymmetric eigenfunctions (translational modes) (a) for the one-pole coalescent steady state, $\psi_{1}^{1, a}$, at $\gamma=3$; (b) for the two-pole coalescent steady state, $\psi_{1}^{2, a}$, at $\gamma=7$; (c) for the three-pole coalescent steady state, $\psi_{1}^{3, a}$, at $\gamma=23$; (d) for the six-pole coalescent steady state, $\psi_{1}^{6, a}$, at $\gamma=26$.

was reported to be unstable. This conclusion was based on the computed growth rates, which were found to be $2 \tilde{\lambda}=0.647 \cdot 10^{-3}$ for case (d), $2 \tilde{\lambda}=2.13 \cdot 10^{-3}$ for case (e), and $2 \tilde{\lambda}=9.409 \cdot 10^{-3}$ for case (f). It was also pointed out by the authors that, in each of these three cases, only one nontrivial eigenmode was found. The shapes of these eigenmodes were similar to each other and to the shapes of the eigenmodes (associated with $\tilde{\lambda}=0$ ) found in the three cases (a)-(c) examined in [5] and discussed above. We first point out that the values of $\gamma$ in cases (e) and (f) correspond exactly to the bifurcation points $\gamma_{6}$ and $\gamma_{8}$. In case (f) the value of $\gamma$ is within the interval $I_{10}$. The steady solutions in all three cases are therefore neutrally stable. The results of the numerical computations of [8] do not agree with our exact solutions in this case. Our exact solutions show that for the first two cases, (d) and (e), the largest eigenvalue is a zero eigenvalue of multiplicity three; one of the eigenvalues is the first antisymmetric eigenvalue of type I, $\lambda_{1}^{N, a} \equiv 0$, and the other is the double eigenvalue $\lambda_{N+1}^{N}$ (for $N=6,8$ ) of type II, which vanishes at exactly $\gamma=26$ and $\gamma=34$. For the last case (f), the largest eigenvalue is the first antisymmetric eigenvalue of type $\mathrm{I}$, $\lambda_{1}^{N, a} \equiv 0$, and it is a simple eigenvalue. Thus, at least one of the eigenmodes is the antisymmetric eigenfunction that corresponds to the translational mode discussed 
above. The shape of these eigenmodes are expected to be similar, and similar to those shown in Figure 4.1 (a)-(c). Now, however, the peaks near the boundaries would be sharper, as expected, because of the larger values of $N$ considered. We have included the eigenmode corresponding the case (d), which exhibits this behavior, in Figure 4.1 (d). From the striking similarity between the eigenmodes calculated in [8] in the three cases just discussed one may have anticipated that $\tilde{\lambda}$ must be zero, as it actually is. There could be several reasons that led to the small positive values of $\tilde{\lambda}$ obtained in the calculations, instead of exact zeroes. In the computations the growth rate was determined from $\tilde{\lambda}=\lim _{t \rightarrow \infty}\left\{\frac{1}{2 t} \ln R(t)\right\}$ with $R(t)=\int_{-L}^{L}(\psi(x, t))^{2} d x$. For neutrally stable states, $\tilde{\lambda}=0$, a slight imperfection in the calculated value of $R(t)$, resulting, for example, from using an insufficiently small number of Fourier modes, can lead to the erroneous small growth rates. Another possible reason could be linked to the high degeneracy (multiplicity three), and therefore high sensitivity to perturbations, of the zero-eigenvalue in cases (d) and (e). In the last case (f), although the value of $\gamma$ is not at one of the bifurcation points, the value is sufficiently small, requiring a large number of harmonics to properly resolve the solution of the PDE by means of spectral methods. As we have already pointed out the reason lies in the fact that the eigenfunction profiles in $k$-space representation become too flat (especially eigenfunctions of type I) as $\gamma$ increases.

Apart from resolving the controversy between the results of $[6,7]$ and $[5,8]$, the simple exact structure of the spectrum that we have derived sheds light on the behavior of the solutions of the linearized PDE (I-4.3) and, to some extent, on the solutions of the nonlinear evolution equation (1.1). For example, it is possible to examine the departure of the solution from an equilibrium state and its evolution to a different state if the latter exists. To this end we examine in detail a case which was also treated in [5]. We consider an interval of length $L=23 \pi$ (corresponding to $\gamma=23 / 2$ ) and assume that the initial condition is the one-pole coalescent steady state. We know that the one-pole solution is unstable for this value $\gamma$. The linearly most unstable mode at this point (see Figure 2.2) is the third eigenvalue $\lambda_{3}^{1}$, which can be evaluated from (1.4), with $\gamma=23 / 2, N=1$, and $S=2$, to be $\lambda_{3}^{1}=7 / 23$. To translate this value to $\tilde{\lambda}$ used in [5], we divide the former by $\gamma$ and find the exact value of the growth rate $2 \tilde{\lambda}=2 \lambda_{3}^{1} / \gamma=28 / 529 \approx 0.05293$. The calculations in [5] led to $2 \tilde{\lambda}=0.052914$, i.e., accurate up to the fourth digit. It was also noted in [5] that two eigenmodes, one symmetric and one antisymmetric, correspond to that $\tilde{\lambda}$. The two modes are obviously a consequence of the fact that $\lambda_{3}^{1}$ is of type II (of multiplicity two) so that there is one symmetric and one antisymmetric eigenfunction associated with it. Employing the exact formulas for eigenfunctions given in subsection 4.3 of Part I we have plotted in Figure 4.2 the two exact eigenmodes, which show that the results in [5, Figure 6] are well within computational errors. At $\gamma=23 / 2$, one sees from Figure 2.2 that there are three pairs of unstable directions in phase space. The pair of the most unstable eigenmodes is the one associated with $\lambda_{3}^{1}$ which should, therefore, be seen in the initial stage of the evolution of the solution. As suggested in [5], the two-dimensionality of the eigenspace explains the observation (from the numerical simulation) that, starting with a coalescent one-pole state, two additional poles appear simultaneously and the solution of the nonlinear PDE (1.1) jumps to a noncoalescent three-pole solution. Since the noncoalescent state is unstable the poles eventually align themselves into a three-pole coalescent state. A similar development occurs in the evolution of wrinkles on a nominally curved flame (see, e.g., $[9,10]$ ). It has been observed that when one starts with a one-pole coalescent state as an 

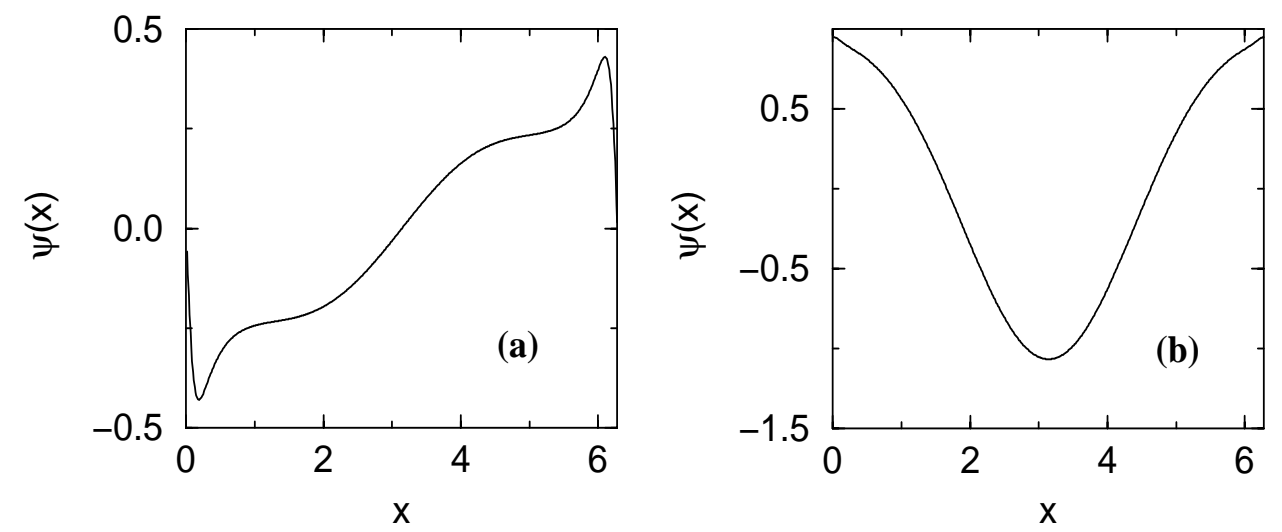

FIG. 4.2. $x$-space representation of the third eigenfunctions for the one-pole coalescent steady state at $\gamma=23 / 2$ : (a) antisymmetric, $\psi_{3}^{1, a}(x)$; (b) antisymmetric, $\psi_{3}^{1, s}(x)$.

initial condition, the solution jumps, after some time, to a three-pole coalescent state and then, after another interval of time, to a five-pole coalescent state, and so on. In this case the parameter $\gamma$, proportional to the radius of curvature of the flame front, increases continuously in time. Intuitively, as the flame grows outwards, its radius increases so that the domain size $L$ and, therefore, the value of $\gamma$ increase. When $\gamma$ reaches the value $\gamma_{N}$, the largest eigenvalue that crosses $\gamma$-axis is of type II (of multiplicity two) and the eigenspace associated with it is two-dimensional. As a consequence there are always two new modes that start growing.

We point out that in this paper, and starting with subsection 4.2 in Part I, we have only considered explicitly the stability of solutions with $r=1$. This is because $N$-pole coalescent steady states with $r>1$ appeared to be always unstable. This was verified by solving the eigenvalue problem for the system (I-4.6) numerically, using the techniques illustrated in section 5 of Part I. This result is in agreement with the conclusions drawn in [5].

In this work we have confined our attention to the stability of coalescent steady states, showing that, for any value of $\gamma$ the steady coalescent $N_{0}$-pole solution is (apart from the trivial translational mode) asymptotically stable. As directly follows from (I-3.13) and (I-3.24), $N_{0} \leq N_{\max }$ and, with the exception of the case $N_{0}=1$, the strict inequality holds. This implies that there could be other, noncoalescent, steady pole solutions of the system (I-3.6). Their existence and stability is an important question that remains to be addressed.

The results of this work provide all the information needed to understand the dynamics of the linearized evolution equation (I-4.3) or, equivalently, of the nonlinear PDE (1.1) for initial conditions that are sufficiently near a coalescent steady state. A question of interest is the evolution of the flame front from arbitrary initial data. Whether, for a given value of the parameter $\gamma$, the long time behavior of the solution of (1.1) is the steady coalescent $N_{0}$-pole solution state depends of course on the domain of attraction of this state. For initial conditions within this domain the solution will eventually converge to the $N_{0}$-pole solution; otherwise a different, possibly noncoalescent state could be attained or the solution could continuously vary in time without reaching a steady state. The numerical results on the flame evolution starting with arbitrary initial conditions reported in $[5,8]$ seem to suggest that the long time 
behavior may depend on the value of the parameter $\gamma$ (or the size of the domain of integration $L$ ). However, the numerics leading to this conclusion is likely to be affected by the deficiencies similar to the ones leading to the erroneous results regarding linear stability. In summary, the nonlinear stability and dynamics still remain an important open question.

\section{REFERENCES}

[1] D. Vaynblat and M. Matalon, Stability of pole solutions for planar propagating flames: I. Exact eigenvalues and eigenfunctions, SIAM J. Appl. Math, 60 (2000), pp. 679-702.

[2] G. I. Sivashinsky, Nonlinear analysis of hydrodynamic instability in laminar flames-I. Derivation of basic equations, Acta Astronautica, 4 (1977), pp. 1177-1206.

[3] G. Darrieus, Propagation d'un front de flamme, Congres de Mecanique Appliquee, 1938.

[4] L. D. Landau, On the theory of slow combustion, Acta Physicochimica, USSR, 19 (1944), pp. $77-85$.

[5] O. Rahibe, N. Aubry, And G. I. Sivashinsky, Stability of pole solutions for planar propagating flames, Phys. Rev. E, 54 (1996), pp. 4958-4972.

[6] O. Kupervasser, Z. Olami, and I. Procaccia, Geometry of developing flame fronts: Analysis with pole decomposition, Phys. Rev. Lett., 76 (1996), pp. 146-149.

[7] Z. Olami, B. Galanti, O. Kupervasser, and I. Procaccia, Random noise and pole dynamics in unstable front propagation, Phys. Rev. E, 55 (1997), pp. 2649-2663.

[8] O. Rahibe, N. Aubry, And G. I. Sivashinsky, Intability of pole solutions for planar propagating flames in sufficiently large domains, Combust. Theory Modelling, 2 (1998), pp. $19-41$.

[9] G. Joulin, Nonlinear hydrodynamic instability of expanding flames: Intrinsic dynamics, Phys. Rev. E, 50 (1994), pp. 2030-2047.

[10] O. Rahibe, N. Aubry, G. I. Sivashinsky, and R. Lima, Formation of wrinkles in outwardly propagating flames, Phys. Rev. E, 52 (1995), pp. 3675-3686. 